\title{
A TLR/AKT/Fox03 immune tolerance-like pathway disrupts the repair capacity of oligodendrocyte progenitors
}

\author{
Taasin Srivastava, ${ }^{1}$ Parham Diba, ${ }^{1}$ Justin M. Dean, ${ }^{1}$ Fatima Banine, ${ }^{2}$ Daniel Shaver, ${ }^{1}$ Matthew Hagen, ${ }^{1}$ Xi Gong, ${ }^{1}$ Weiping Su, ${ }^{2}$ Ben Emery, ${ }^{3}$ \\ Daniel L. Marks, ${ }^{1}$ Edward N. Harris, ${ }^{4}$ Bruce Baggenstoss, ${ }^{5}$ Paul H. Weigel, ${ }^{5}$ Larry S. Sherman, ${ }^{2,6}$ and Stephen A. Back ${ }^{1,3}$ \\ 'Department of Pediatrics, Oregon Health \& Science University (OHSU), Portland, Oregon, USA. ²Division of Neuroscience, Oregon National Primate Research Center, Beaverton, Oregon, USA \\ ${ }^{3}$ Department of Neurology, OHSU, Portland, Oregon, USA. ${ }^{4}$ Department of Biochemistry, University of Nebraska-Lincoln, Lincoln, Nebraska, USA. ${ }^{5}$ Department of Biochemistry and Molecular Biology, \\ University of Oklahoma Health Sciences Center, Oklahoma City, Oklahoma, USA. ${ }^{6}$ Department of Cell, Developmental and Cancer Biology, OHSU, Portland, Oregon, USA
}

Cerebral white matter injury (WMI) persistently disrupts myelin regeneration by oligodendrocyte progenitor cells (OPCs). We identified a specific bioactive hyaluronan fragment (bHAf) that downregulates myelin gene expression and chronically blocks OPC maturation and myelination via a tolerance-like mechanism that dysregulates pro-myelination signaling via AKT. Desensitization of AKT occurs via TLR4 but not TLR2 or CD44. OPC differentiation was selectively blocked by bHAf in a maturation-dependent fashion at the late OPC (preOL) stage by a noncanonical TLR4/TRIF pathway that induced persistent activation of the FoxO3 transcription factor downstream of AKT. Activated Fox03 selectively localized to oligodendrocyte lineage cells in white matter lesions from human preterm neonates and adults with multiple sclerosis. FoxO3 constraint of OPC maturation was bHAf dependent, and involved interactions at the FoxO3 and MBP promoters with the chromatin remodeling factor Brg1 and the transcription factor Olig2, which regulate OPC differentiation. WMI has adapted an immune tolerance-like mechanism whereby persistent engagement of TLR4 by bHAf promotes an OPC niche at the expense of myelination by engaging a FoxO3 signaling pathway that chronically constrains OPC differentiation.

\section{Introduction}

Immune tolerance (IT) is a transient state in cells of the innate immune system where repeated exposure to pathogen-associated molecular patterns (PAMPs) or damage-associated molecular patterns (DAMPs) renders cells unresponsive to further PAMP/ DAMP exposure $(1,2)$. Removal of the PAMP/DAMP recalibrates the system with restoration of immune responses. An initial excessive inflammatory response via TLRs can induce a tolerant state (1). IT has evolved to prevent excessive immune system mediated self-injury in response to infection, inflammation, or tissue injury. However, some bacteria exploit IT to evade immune system surveillance. Repeated exposure to bacterial endotoxin chronically suppresses host proinflammatory immune responses, which causes bacteria to overwhelm and kill the host.

In the central nervous system, the megadalton form of hyaluronan (MDa HA) is a major component of the extracellular matrix (ECM) (3). MDa HA accumulates in demyelinating lesions, where it promotes myelination failure by blocking maturation of oligodendrocyte progenitor cells (OPCs) (4), which would otherwise mature into myelinating oligodendrocytes (5). HA fragments (HAf) are generated when white matter injury (WMI) triggers enhanced expression of hyaluronidases that depolymerize $\mathrm{MDa}$

Conflict of interest: The authors have declared that no conflict of interest exists Submitted: March 22, 2017; Accepted: February 28, 2018.

Reference information: J Clin Invest. 2018;128(5):2025-2041.

https://doi.org/10.1172/JCI94158.
HA $(6,7)$. In conditions such as sterile lung injury and wound healing, ECM breakdown results in release of proinflammatory HAf (8), which may function as an endogenous DAMP to regulate proinflammation via receptors that include CD44, TLR2, and TLR4 (8). It is unclear, however, whether HAf may also function as a DAMP in chronic WMI to disrupt myelination via a tolerance-like mechanism. OPCs express TLRs, but their stimulation does not promote proinflammatory cytokine release (9). Although, $\mathrm{I} \kappa \mathrm{K} / \mathrm{NF}-\kappa \mathrm{B}$ signaling is the main proinflammatory pathway associated with TLR activation, it does not regulate developmental myelination or remyelination (10). Despite evidence that HAf may mediate adult myelination failure by utilizing a proinflammatory signaling pathway (6), the proinflammatory actions of HAf have been recently questioned and attributed to bacterial endotoxin contamination (11). Consequently, the mechanistic roles for $\mathrm{MDa}$ HA and/or HAf in regulating OPC differentiation in chronic WMI remain poorly defined.

WMI in preterm human infants causes arrested maturation of late OPCs (preOLs) in chronic lesions that contributes to cerebral palsy and chronic neurobehavioral disabilities (12). We report here that neonatal WMI results in ECM breakdown and a persistent reduction in MDa HA. To define potential roles of HAf in chronic neonatal myelination failure, we generated endotoxin-free HAf of narrowly restricted sizes. We identified a specific bioactive HAf (bHAf) of approximately $200 \mathrm{kDa}$ that blocked OPC maturation cell autonomously. Sustained exposure of OPCs to bHAf led to desensitization of AKT, a potent 
A

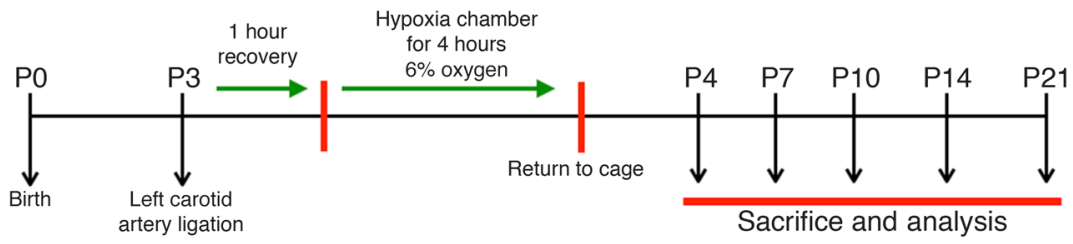

B
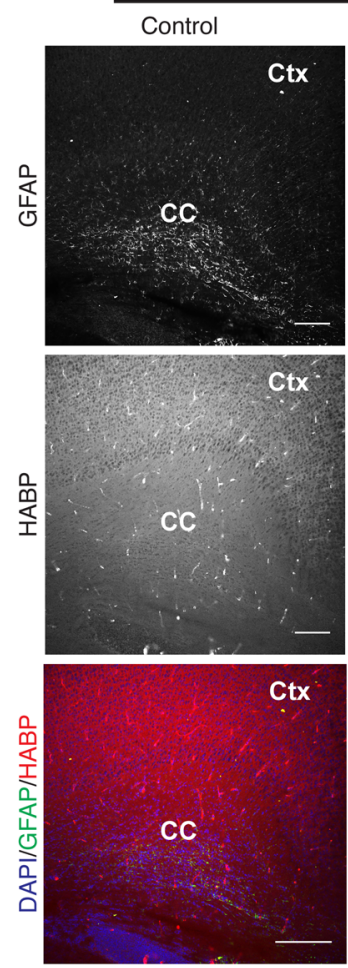

$\mathrm{P} 4$
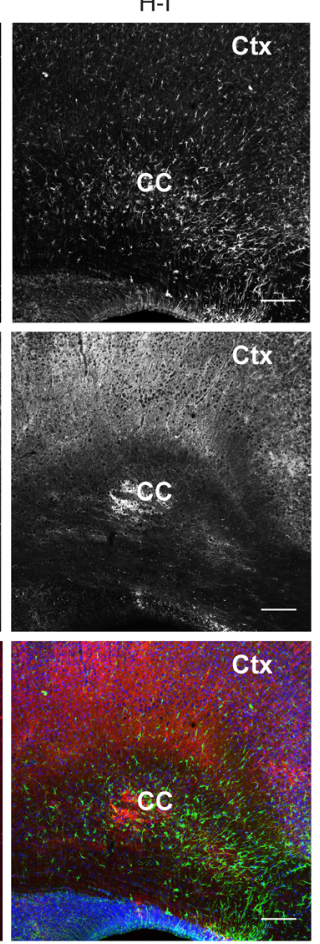

C
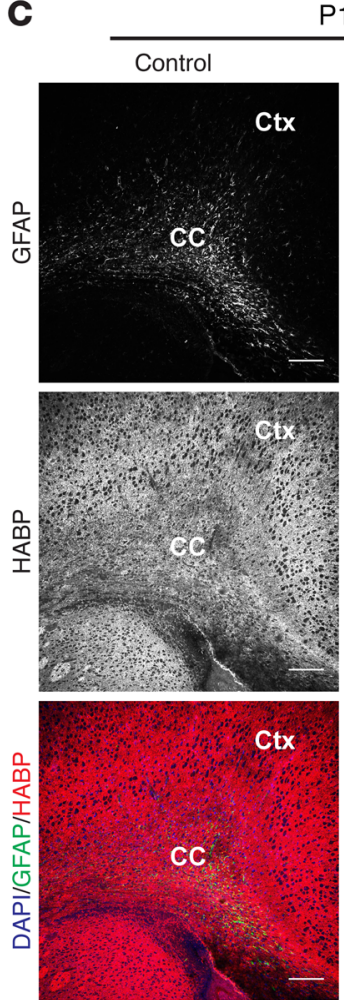

$\mathrm{P} 14$
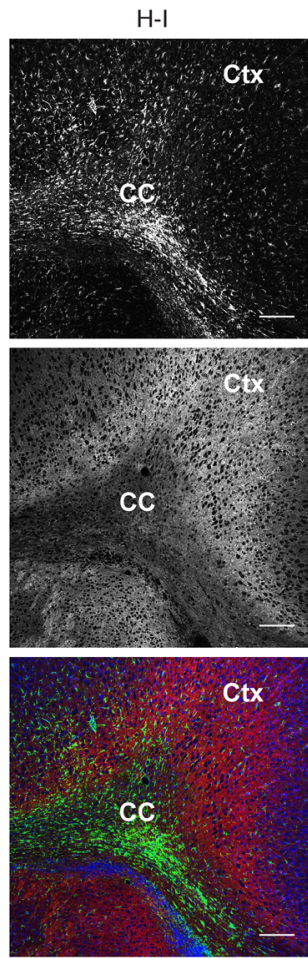

D

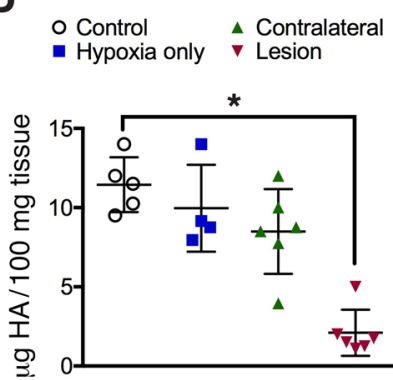

E

$\begin{array}{rcccc}\text { MDa HA } & + & + & + & + \\ \text { Boiled H-l lysate } & - & + & - & - \\ \text { H-l lysate } & - & - & + & + \\ \text { Deferoxamine } & - & - & - & +\end{array}$

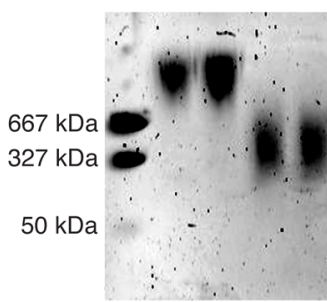

Figure 1. Neonatal rat H-I WM lesions deplete MDa HA. (A) Schematic diagram of the H-I strategy and postnatal ages for tissue analysis. (B and C) Representative images of the supracallosal WM of the corpus callosum (CC) and overlying cortex (Ctx) in control and $\mathrm{H}-\mathrm{I}$ samples at P4 (B) and P14 (C) stained for GFAP and HABP. Diffuse reduction in MDa HA persisted until P14 (C). (D) Recovery of total HA from WM lesions after H-I. Comparison of true controls (Control), hypoxic controls (Hypoxia only), and $\mathrm{H}$-I group (Lesion) versus contralateral hypoxic hemisphere (Contralateral). Only the lesion group had a significant reduction in HA recovery. (E) Incubation of MDa HA with the lesion lysate generated HAf below $\sim 650 \mathrm{kDa}$. Lesion-lysate activity was sensitive to heat inactivation but insensitive to deferoxamine $(50 \mu \mathrm{M})$. B and C: control $n=2 ; \mathrm{H}-\mathrm{I} n=4$ animals for each age group (P4 and P14). D: $n=6$ (H-I), $n=5$ (control), and $n=4$ (hypoxia) animals (P7). E: $n=4$ separate experiments on 4 different animals at P4 after $\mathrm{H}-\mathrm{I}$ at P3; one representative experiment is shown. ${ }^{*} P<0.05$ by ANOVA. Mean \pm SD. Scale bars: $300 \mu \mathrm{m}$ (B and $\mathbf{C}$ ).

pro-myelination signal (13). This tolerance-like action of bHAf was mediated through TLR4 but not via CD44 or TLR2. As in TLR4-mediated IT, bHAf's influence on myelination was reversible when MDa HA depolymerization was attenuated or bHAf was removed. AKT desensitization was similarly reversible in vivo in neonatal WMI. Moreover, bHAf actions were mediated via a noncanonical TRIF-dependent pathway, also involved in IT, rather than the canonical MyD88 arm of TLR4 signaling. AKT desensitization resulted in maturation-dependent activation of the FoxO3 transcription factor (TF), which selectively constrained preOL maturation in a bHAf-dependent fashion. A role for activated $\mathrm{FoxO} 3$ in human myelination failure was supported by selective localization of nuclear $\mathrm{FoxO} 3$ to OPCs in human preterm WMI and multiple sclerosis (MS) plaques. bHAf-mediated OPC maturation arrest appears to be regulated at the $\mathrm{FoxO} 3$ and myelin basic protein (MBP) promot- ers by interactions of FoxO3 with the chromatin-remodeling factor Brg1 and the TF Olig2, which are involved in control of OPC differentiation (14-16). Hence, central myelination failure is regulated by a noncanonical TLR4/AKT/FoxO3 signaling pathway utilized by bHAf to induce a tolerance-like state that selectively constrains OPC maturation and myelination.

\section{Results}

Neonatal hypoxic-ischemic WMI promotes MDa HA depolymerization. To investigate the status of MDa HA in chronic neonatal WMI, we employed our preterm-equivalent rat hypoxia-ischemia (H-I) model, which generates myelination failure and replicates key features of human WMI (Figure 1A) (17). MDa HA turnover in the ECM after H-I was visualized with a biotinylated HA-binding protein (HABP) and costained with glial fibrillary acidic protein (GFAP) as a marker of WMI. Unlike in age-matched unin- 
jured control white matter (WM), diffuse astrocyte activation was observed within 24 hours after $\mathrm{H}-\mathrm{I}$ in the corpus callosum at P4 (Figure 1B). HABP staining revealed uniform distribution of $\mathrm{MDa}$ HA in control gray and white matter at P4 (Figure 1B and Supplemental Figure 1A; supplemental material available online with this article; https://doi.org/10.1172/JCI94158DS1). However, MDa HA staining was markedly reduced throughout early WMI (Figure 1B). In contrast to controls at P14, chronic WMI displayed extensive reactive astrogliosis and a persistent diffuse reduction in MDa HA (Figure 1C).

To obtain a quantitative estimate of MDa HA depolymerization in neonatal WMI, we isolated HA from neonatal brain tissue for analysis by size exclusion chromatography coupled to multi-angle light scattering (SEC-MALS). Lesions showed a significant pronounced decrease in MDa HA content relative to true controls (Figure 1D). There was no significant reduction in MDa HA recovery from the contralateral hypoxic hemisphere or from exposure to hypoxia alone (Figure 1D).

We next examined expression of enzymes that regulate HA levels in neonatal WMI. We found no changes in expression of HA synthases 1-3 (Supplemental Figure 1B), which generate different HA sizes (8). De-polymerization of MDa HA by hyaluronidases can generate large (300 kDa-1 MDa), intermediate (20-300 kDa), or small $(<20 \mathrm{kDa}) \mathrm{HAf}$, which regulate distinct signaling pathways (18). Previous reports implicated MDa HA depolymerization by the hyaluronidase $\mathrm{PH} 2 \mathrm{O}$ in regulation of re-myelination in adult models of WMI (refs. 6, 7; but also see ref. 19). We did not detect $\mathrm{PH} 20$ expression in neonatal rat brain (Supplemental Figure 1B). No changes in Hyal2 expression occurred after H-I, but there was a significant decrease in expression of Hyal1, which generates HAf oligomers (18). In contrast, expression of Tmem2, a recently described membrane-associated hyaluronidase (20), was significantly elevated after $\mathrm{H}-\mathrm{I}$.

We next determined whether neonatal WMI had an enzymatic activity that could generate HAf. When protein lysates made from $\mathrm{H}-\mathrm{I}$ lesions were incubated with exogenous MDa HA, HAf were generated in a broad range below approximately $650 \mathrm{kDa}$ (Figure $1 \mathrm{E})$. However, HAf were not generated from pre-boiled protein lysates (Figure 1E). Since non-enzymatic MDa HA depolymerization may occur by oxidative attack, we coincubated WMI lysates with deferoxamine, because neonatal WM is highly enriched in iron, which may catalyze production of superoxide radicals that nonspecifically generate HAf. Under these conditions, MDa HA depolymerization was still observed (Figure 1E). Hence, we isolated an endogenous activity in response to neonatal WMI whereby MDa HA is cleaved into a range of smaller fragments.

A distinct sized HA ligand is bioactive and mediates OPC maturation arrest. To define a role for MDa HA depolymerization in myelination failure, we first determined whether myelination occurred in our in vitro slice culture model of chronic WMI. This model reproduces key features of neonatal WMI including OPC maturation arrest, which is enhanced by MDa HA (21). Since slice cultures were prepared from P1 animals, myelin was not present. However, between 14 and 21 days in culture, we visualized extensive staining for the juxtaparanodal myelin marker CASPR, consistent with formation of nodes of Ranvier (Figure 2A), and detected numerous neurofilament protein-labeled axons associated with MBP-labeled oligodendrocytes (Supplemental Figure 2A). To confirm de novo progressive myelin generation, we undertook ultrastructural studies that identified axons wrapped with multilamellar myelin sheaths (Figure 2B).

In contrast to vehicle-treated slices, which displayed robust myelination of the corpus callosum, slices incubated with MDa HA until 21 days in vitro (DIV21) displayed a pronounced reduction in myelinated axons (Figure 2C). Myelination failure was not related to decreased OPC survival, since MDa HA treatment did not enhance OPC degeneration or decrease OPC density (Supplemental Figure 2, B-D). Notably, myelination comparable to that in vehicle-treated controls was promoted by coincubation of slices with MDa HA and VCPAL, a potent hyaluronidase inhibitor (22) (Figure 2C). We confirmed that the effect of VCPAL was cell autonomous. Consistent with prior studies $(4,6,19)$, incubation of differentiating primary OPC cultures with MDa HA induced a significant decrease in the percentage of mature $\mathrm{MBP}^{+} \mathrm{OLs}$, which was significantly reversed by coincubation of primary OPCs with MDa HA and VCPAL (Supplemental Figure 2E). Hence, the disruption of myelination by MDa HA appeared to be cell autonomous and mediated by MDa HA depolymerization induced by hyaluronidase activity.

Since we had identified an endogenous activity in neonatal WMI lysates that generated a range of smaller HAf (Figure 1E), we performed an in vitro screen for a bioactive size range of HAf that blocks OPC maturation. We first screened polydispersed fractions of HAf, which contained a continuum of HA sizes, to define size ranges that blocked OPC differentiation. HAf sizes between 175 and $300 \mathrm{kDa}$ attenuated OPC maturation, whereas small (5-20 kDa) HAf ranges lacked activity (Figure 2D). To more narrowly define the size of bHAf, we prepared endotoxin-free monodisperse HAf $(40,106,210$, and $357 \mathrm{kDa})$ by SEC fractionation of polydispersed fractions of HA (23) and screened them in slice cultures. OPC maturation was not blocked by 40-, 106-, or 357kDa HAf (Figure 2E). In contrast, treatment with 210-kDa HAf decreased OPC maturation (Figure 2E). We confirmed that bHAf blocked maturation of primary OPC cultures in a cell-autonomous manner (Figure 2F). Moreover, after sonication of MDa HA and bHAf, these preparations failed to block OPC maturation (Supplemental Figure 2F). Notably, bHAf persistently blocked OPC maturation and myelination in slice cultures up to DIV21 (Figure 2C and Supplemental Figure 2G).

We next determined whether the effect of bHAf on OPC maturation was concentration dependent or altered by coincubation with HAf of different sizes. Concentrations of 100-200 nM attenuated OPC maturation in slices, whereas lower or higher concentrations lacked activity, which suggested a narrow critical concentration range for biological activity (Figure 2G). To attempt to out-compete and block bHAf activity, we cotreated slices with $100 \mathrm{nM}$ bHAf and equimolar concentrations of inactive HAf (40, 106 , or $357 \mathrm{kDa})$. Neither HAf sizes smaller nor those larger than $210 \mathrm{kDa}$ disrupted bHAf activity, supporting the hypothesis that bHAf selectively blocks OPC maturation (Figure 2H). Hence, our findings indicate that attenuation of OPC maturation in the lesion microenvironment is dependent on generation of an effective concentration of bHAf by hyaluronidase activity, and that bHAf with a distinct size of about $200 \mathrm{kDa}$ is required. 
A

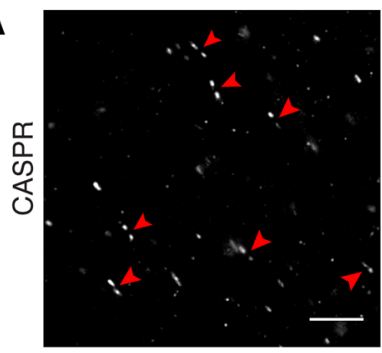

B

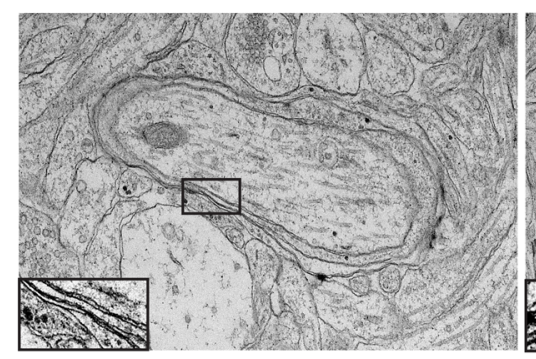

DIV21
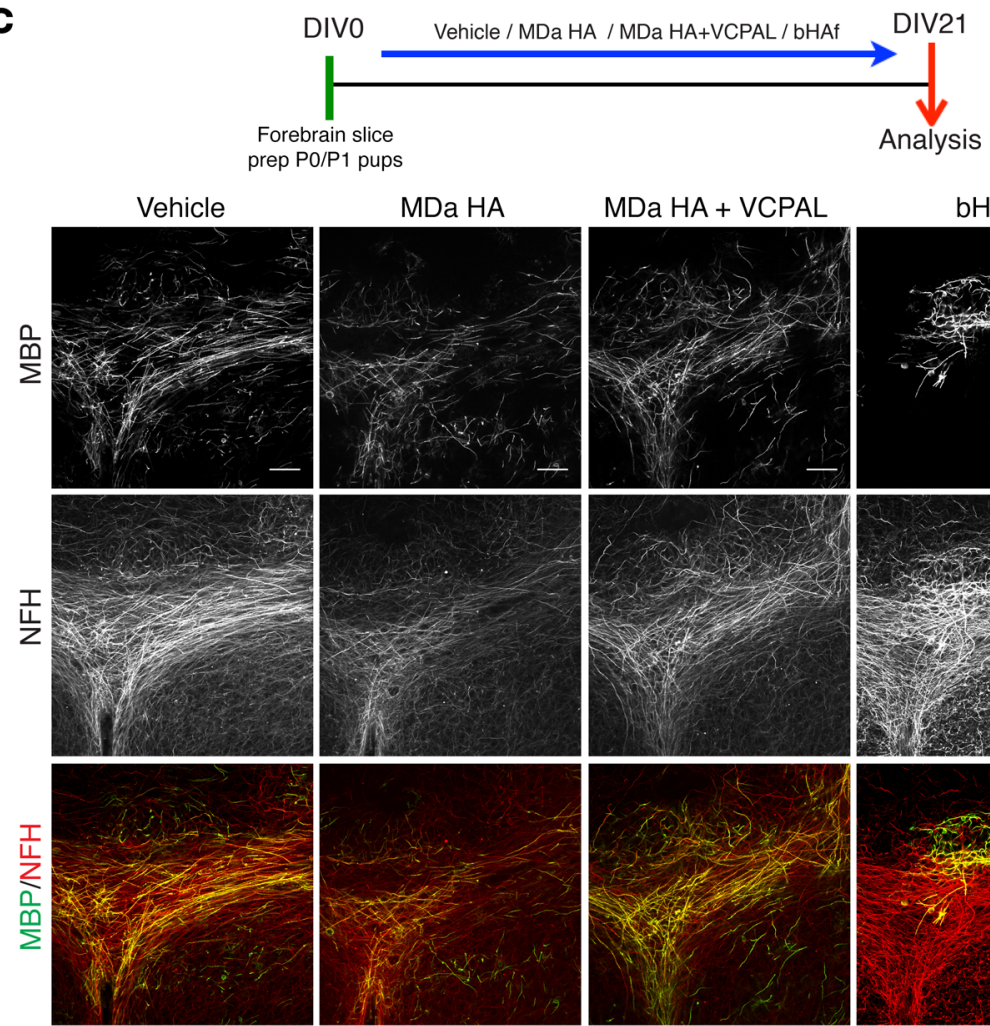

E

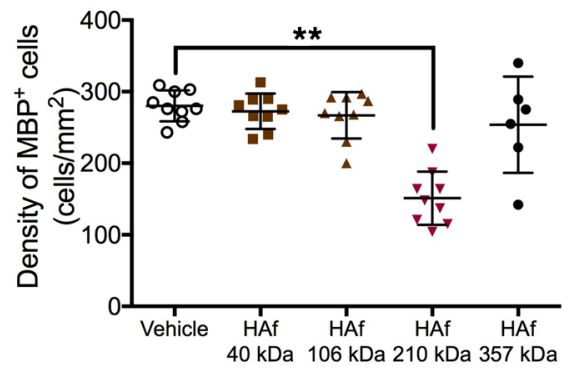

G

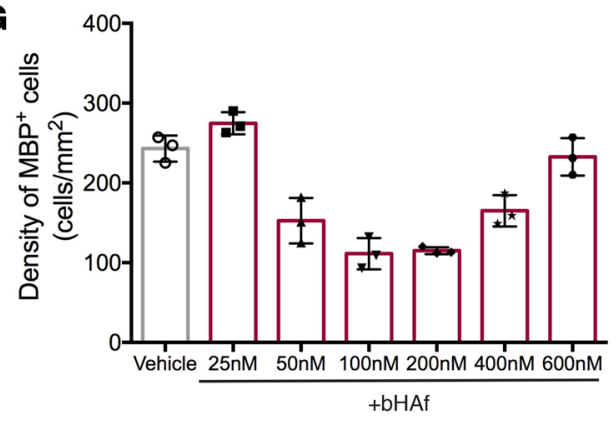

MDa HA + VCPAL

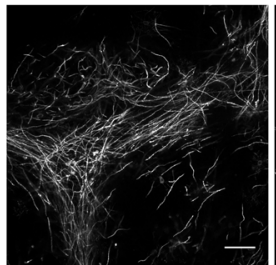

bHAf
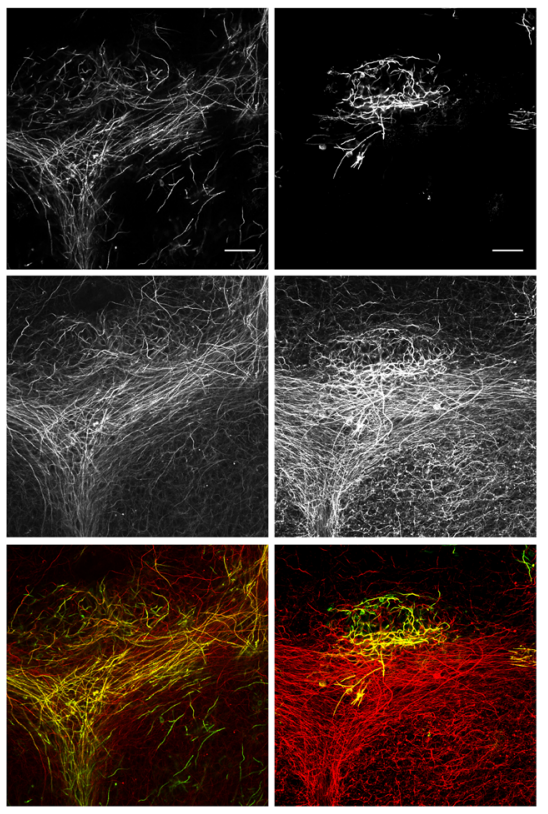

$\mathbf{F}$
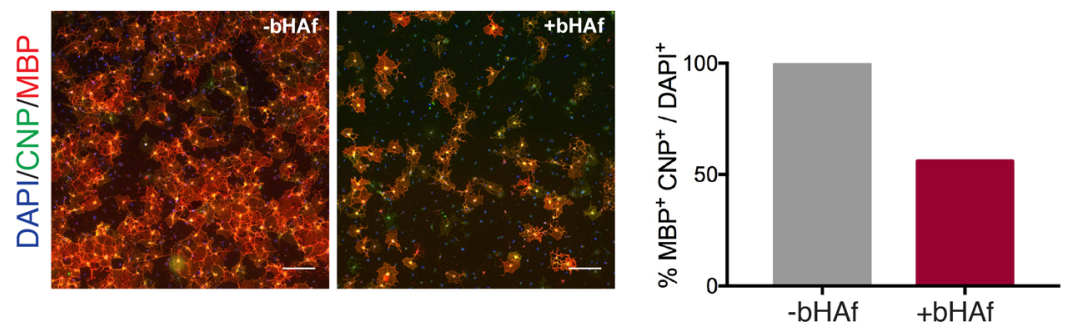

H

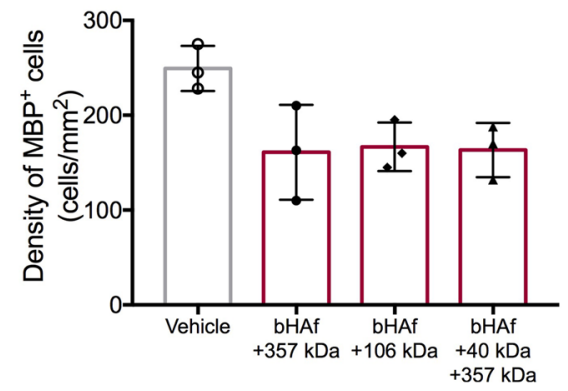


Figure 2. A distinct bHAf blocks OPC maturation. (A-C) Rat slices cultured 21 days display extensive myelination. (A) Nodes of Ranvier identified with CASPR (red arrowheads). (B) Representative ultrastructural images of early (left panel) and late (right panel) myelination and detail of multilamellar myelin sheaths (insets). (C) Schematic of strategy to analyze chronic in vitro myelination. prep, prepared from. Representative images of myelinated axons in callosal WM visualized with MBP and $200 \mathrm{kDa}$ neurofilament $\mathrm{H}$ (NFH) after treatment with DMSO (vehicle), MDa HA (50 nM) with or without VCPAL $(25 \mu \mathrm{M})$, or bHAf (100 nM). (D) 175-300 kDa HAf regulates OPC maturation cell autonomously. Primary OPCs cultured 4 days under pro-differentiation conditions stained for OPCs (PDGFR $\alpha$, green), OLs (MBP, red), and DAPI (blue). Cultures treated with PBS (vehicle), MDa HA (50 nM), HAf 175-300 kDa (500 nM), or HAf 5-20 kDa (500 nM). Quantification of total OPCs and OLs. (E) MBP+ OLs in DIV8 slices cultured with PBS (vehicle) or HAf of 40 (100 nM), $106(100 \mathrm{nM}), 210(100 \mathrm{nM})$ or $357 \mathrm{kDa}$ $(100 \mathrm{nM})$. (F) Primary OPCs differentiated 4 days with or without bHAf (500 nM) visualized with cyclic nucleotide phosphodiesterase (CNP), MBP, and DAPI (left). bHAF treatment decreased the percentage of OLs (right). (C) Density of OLs in DIV8 slices cultured with varying bHAf concentrations. (H) OL quantification in DIV8 slices cultured with bHAf (100 nM) and equimolar concentrations of $\mathrm{HAf}$ at 357 or $106 \mathrm{kDa}$ or combined with 357$\mathrm{kDa}$ and 106-kDa HAf (100 nM). A, C, and E: $n=3$ animals/condition from separate litters; 3 slices/treatment condition/animal, 9 slices total. D and $\mathbf{F}: n=2$ separate culture preparations. $\mathbf{G}$ and $\mathbf{H}: n=1$ animal per condition; 3 slices analyzed per treatment condition. ${ }^{*} P<0.05$ by Student's $t$ test; ${ }^{*} P<0.001$ by ANOVA; mean \pm SD. Scale bars: $12 \mu \mathrm{m}, \mathbf{A} ; 600 \mathrm{~nm}, \mathbf{B}$ (insets: original magnification, $\times 33,000) ; 150 \mu \mathrm{m}, \mathbf{C} ; 75 \mu \mathrm{m}, \mathbf{D}$ and $\mathbf{F}$.

bHAf regulates AKT in a tolerance-like manner. HAf-mediated downstream signaling has been linked to regulation of AKT and MAPK signaling pathways (8). MAPK and AKT also have welldocumented roles in CNS myelination (24). Sustained constitutive AKT phosphorylation is linked with enhanced myelination (13). We first determined in slice cultures whether bHAf influences these signaling pathways. bHAf induced AKT phosphorylation at 15 minutes. However, phosphorylation of both T308 and S473, the two residues associated with maximal AKT activation, was not sustained and returned to baseline by 4 hours (Figure $3 \mathrm{~A}$ ). In contrast, MAPK activation, as defined by ERK1/2 phosphorylation status, did not change significantly, even after 4 hours of bHAf treatment (Figure 3A). AKT phosphorylation remained attenuated after a 24-hour exposure to bHAf (Figure 3B) and even at 9 days (Figure 3C).

To determine whether bHAf selectively induced a refractory state of AKT signaling, we incubated slices with brain-derived neurotrophic factor (BDNF), which robustly stimulates AKT activation and protects against cerebral injury during neonatal $\mathrm{H}-\mathrm{I}$ $(25,26)$. In contrast to bHAf, BDNF sustained AKT activation at 4 and 24 hours (Figure 3D). We next hypothesized that if persistent exposure to bHAf causes chronic desensitization of AKT activation, then AKT should remain refractory to costimulation by another potent AKT activator, such as BDNF. Slices were exposed to bHAf or BDNF, followed by restimulation with the same factor (Figure 3E). Slices remained refractory to AKT activation upon restimulation with bHAf. However, restimulation with BDNF induced AKT activation, indicating that initial BDNF treatment does not induce a refractory state that renders subsequent insensitivity to BDNF. Notably, BDNF treatment was unable to induce AKT activation in slices that were pretreated with bHAf. Hence, continuous bHAf exposure promoted sustained desensitization of AKT that could not be overcome by costimulation with BDNF.
We next determined whether chronic hypoxic-ischemic neonatal WMI is also associated with persistent AKT desensitization (Figure 3F). At 24 hours after H-I (P4), AKT phosphorylation at S473 and T308 was reduced versus that of age-matched uninjured controls. Attenuated AKT phosphorylation persisted at P7 and P10. However, at P14 and P21, AKT phosphorylation was comparable to that of controls. We thus hypothesized that if attenuation of AKT phosphorylation was related to myelination delay, recovery of AKT phosphorylation should coincide with the delayed onset of myelination. We quantified MBP staining as a surrogate marker of myelination at P10, P14, and P21 after H-I at P3 (Figure 3G). MBP staining was markedly reduced at $\mathrm{P} 10$, consistent with a reduction in AKT activation in chronic lesions. However, at P14 and P21, the levels of $\mathrm{MBP}$ in the WM and cortex increased significantly, consistent with a progressive partial increase in myelination. Moreover, in resolving WMI at P21, lesions did not display persistent digestion of $\mathrm{MDa}$ HA, but rather had more normalized MDa HA staining (Supplemental Figure 3). Given that H-I-induced AKT inactivation was reversible and coincided with MDa HA accumulation, we hypothesized that bHAf generation during WMI creates a permissive state that negatively influences OPC maturation in a reversible fashion. We first treated slices with MDa HA and then cultured them without MDa HA (Supplemental Figure 4, A and B), which resulted in an $\mathrm{MBP}^{+} \mathrm{OL}$ density comparable to that in controls. Hence, the presence of bHAf in WM lesions is permissive to sustain a tolerance-like state of AKT desensitization, but upon its removal, OPCs remain competent to differentiate.

bHAf signals via TLR4 but not TLR2 or CD44 to mediate OL maturation arrest. Given that TLRs play prominent roles in regulating IT (2) and CD44 can regulate either TLR2 or TLR4 signaling (8), we determined whether these receptors were involved in bHAf signaling. We adapted our rat slice culture model to knockout mice (Figure 4A). We confirmed that treatment of WT cultures with either MDa HA or bHAf blocked OPC maturation (Figure 4B). We next analyzed slices prepared from $\mathrm{CD} 44^{-/-}$pups. MDa HA failed to block OPC maturation, but bHAf significantly decreased the density of $\mathrm{MBP}^{+}$OLs (Figure 4C). Hence, bHAf action was not mediated via CD44, but CD44 appeared to play a role in the recognition and size processing of MDa HA, as previously reported (27).

We next explored a potential role for TLR2 or TLR4 in bHAf actions. As in $\mathrm{CD} 44^{-/-}$mice, MDa HA failed to block OPC maturation but bHAf blocked OPC maturation in TLR2 ${ }^{-/-}$mice (Figure 4D). However, OPC maturation in TLR4 ${ }^{-/-}$mice was insensitive to either MDa HA or bHAf (Figure 4E). We next confirmed that bHAf signals in a cell autonomous manner via TLR4 but not TLR2. The maturation of TLR2 ${ }^{-/-}$primary OPCs was blocked by bHAf, but OPCs from TLR $4^{-/-}$pups fully differentiated in the presence of bHAf (Supplemental Figure 5). Hence, TLR4 was required for mediation of the inhibitory effects of bHAf on OPC maturation. Given the previously reported complex signaling crosstalk between TLR2 and TLR4 in the brain (28), we determined whether this requirement for TLR4 might be lost with TLR2 deletion by analyzing slices from TLR2-/TLR4 ${ }^{-/-}$mice. These animals were insensitive to either MDa HA or bHAf treatment, which further supported that even with TLR2 deletion, TLR4 deletion still blocked the actions of bHAf (Figure 4F). Collectively, these observations support that bHAf signals via TLR4 to block OPC maturation during chronic WMI. 
A
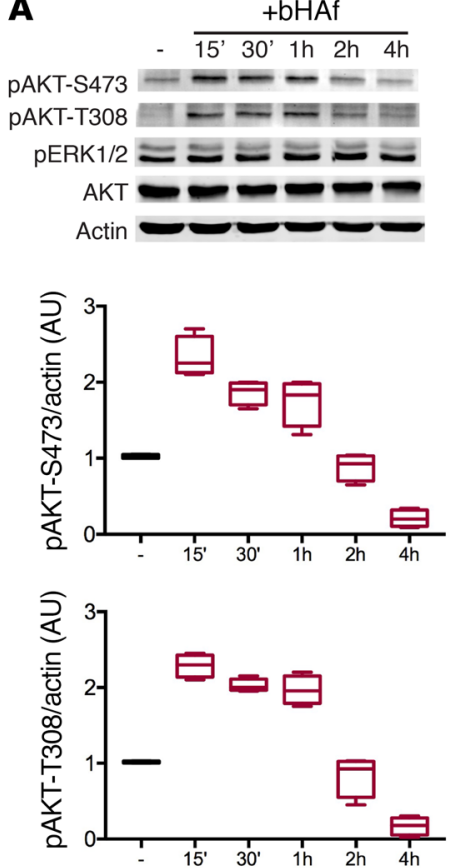

D

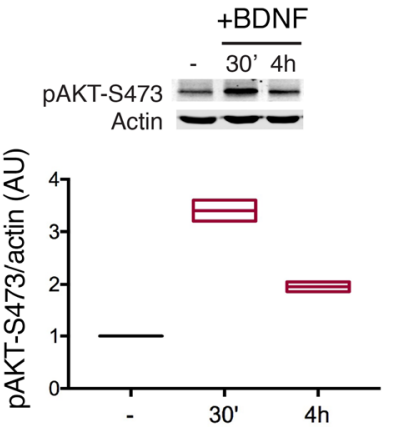

E

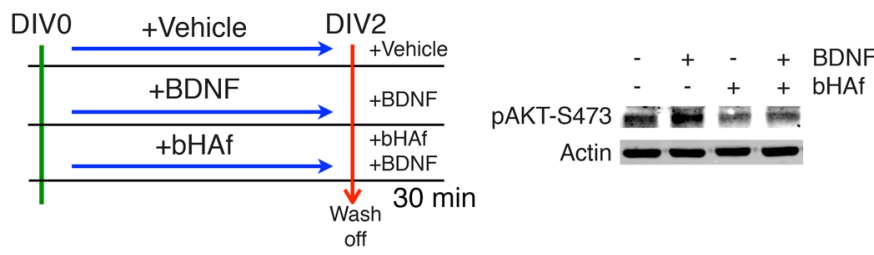

B
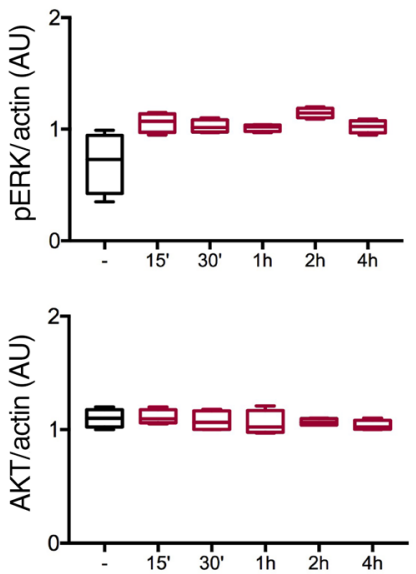

C
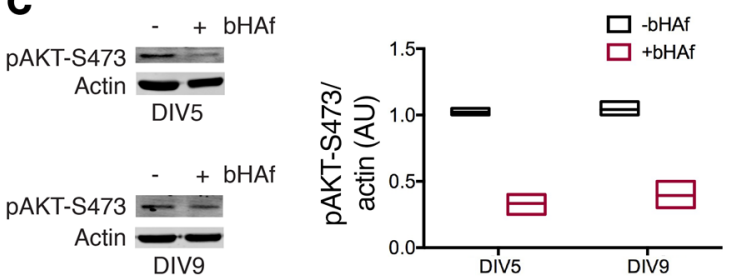

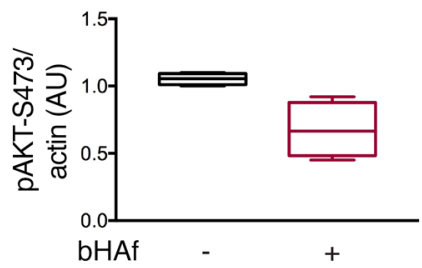

int
$\mathbf{F}$

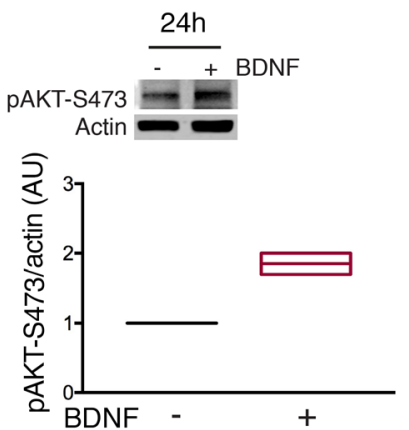
pAKT-S473 C $\frac{\text { C }}{123}$ Actin $\longrightarrow-$ $\mathrm{P} 4$

C $\frac{\mathrm{H}-\mathrm{I}}{123}$ pAKT-S473 $= \pm$
pAKT-T308 $=\cdots$ PAKT-T308 $\div$ $\mathrm{P} 10$

C $\frac{\mathrm{H}-\mathrm{I}}{23}$ pAKT-S473 pAKT-T308 Actin $-\infty$ $\mathrm{P} 21$ Actin -
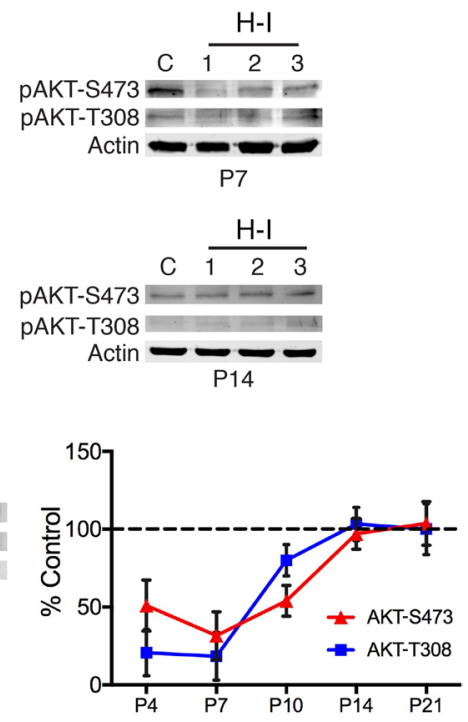

G
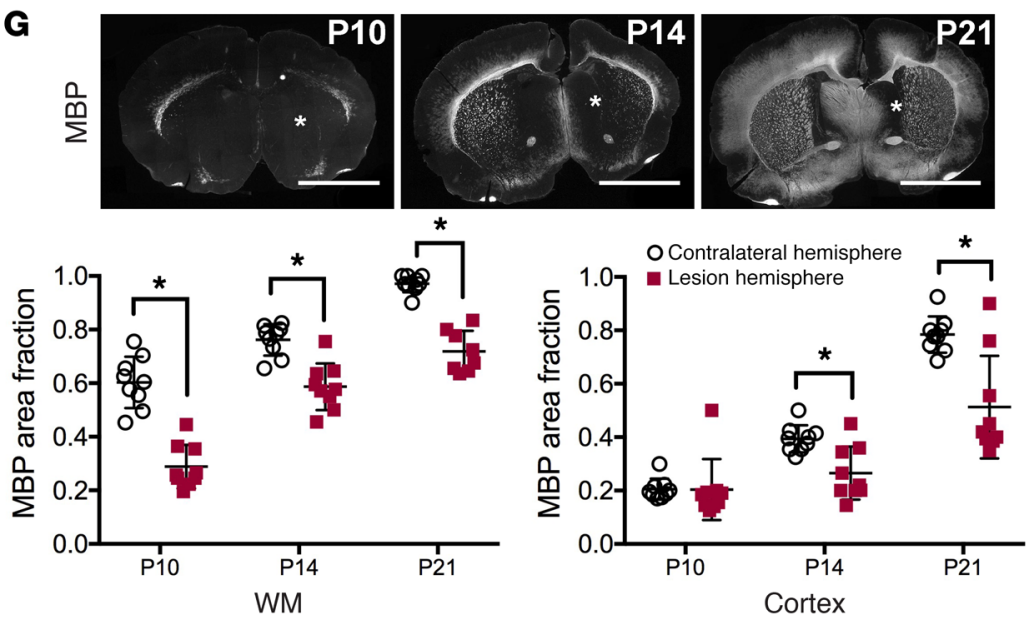
Figure 3. bHAf regulates AKT in a tolerance-like manner. (A-C) bHAf induces persistent AKT dephosphorylation in rat slices treated with bHAf $(100 \mathrm{nM})$. Representative blots and quantification after probing with pAKT-S473, pAKT-T308, pERK1/2, AKT (total AKT), and actin antibodies. Note the transient increase in AKT phosphorylation followed by persistent reduction to control levels at 4 hours (A), 24 hours (B), and 5 or 9 days (C). (D) BDNF $(50 \mathrm{ng} / \mathrm{ml})$ induces persistent AKT phosphorylation at 30 minutes, and 4 or 24 hours. (E) Experimental design to determine whether BDNF can overcome bHAf-mediated AKT desensitization (left); and representative blots (right). (F) Chronic WMI leads to persistent AKT dephosphorylation that normalizes with delayed partial myelination. Representative blots and quantification from 3 rats that underwent $\mathrm{H}-\mathrm{I}$ at $\mathrm{P} 3$ versus control (C), comparing AKT phosphorylation in lesion hemisphere at $\mathrm{P} 4, \mathrm{P} 7$, P10, P14, and P21. Actin was the loading control. (G) Progressive recovery of myelination following WMI. Representative images depicting MBP staining at $\mathrm{P} 10, \mathrm{P} 14$, and $\mathrm{P} 21$ (asterisks indicate $\mathrm{H}-\mathrm{I}$ hemisphere). A-E: $n=3$ independent studies from 3 separate litters; 2 slices/condition. F: $n=3$ animals (control) and $n=6$ animals $(\mathrm{H}-\mathrm{I}) . \mathbf{G}: n=9$ animals/condition. ${ }^{*} P<0.05$ by Student's $t$ test; mean \pm SEM. Scale bars: $600 \mu \mathrm{m}$, G.

bHAf signals via TRIF to regulate AKT. TLR4-mediated tolerance may be regulated at multiple levels via 2 distinct adaptors, MyD88 and TRIF $(1,2)$. We first determined which of these adaptor proteins participates in bHAf signaling (Figure $5 \mathrm{~A}$ ). Both $\mathrm{MDa} \mathrm{HA}$ and bHAf caused a significant decrease in $\mathrm{MBP}^{+} \mathrm{OLs}$ in

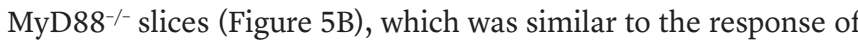
WT mice to bHAf (Figure 4B). Hence, the actions of neither MDa HA nor bHAf appeared to be mediated via the canonical MyD88 signaling pathway. However, when we analyzed $\mathrm{TRIF}^{-/-}$pups, neither bHAf nor MDa HA blocked OPC maturation (Figure 5C). Hence, bHAf blocked OPC maturation through TLR4 via a noncanonical signaling pathway that utilized the TRIF adaptor protein.

We next determined whether TRIF is involved in bHAfmediated AKT activation. We treated slices from $\mathrm{TRIF}^{-/}$or WT pups with bHAf and determined AKT phosphorylation status. Treatment with bHAf for 30 minutes did not cause significant changes in AKT phosphorylation in $\mathrm{TRIF}^{-/-}$cultures (Figure 5D), whereas AKT was phosphorylated in WT cultures (Supplemental Figure 6A). We also analyzed AKT activation after 24 hours of bHAf treatment in $\mathrm{TRIF}^{-/-}$(Figure 5E) and TLR4 ${ }^{-/-}$(Figure 5F) slices and similarly found no change in AKT phosphorylation status, which further supported that bHAf signals via the TLR4/TRIF pathway.

We then determined whether negative regulators of TLR 4 signaling act through a complex with TRIF. We examined the role of TRAF6, an E3 ligase, which is a member of the TRIF signalosome complex that plays a role in AKT ubiquitination and activation $(2,29)$. Proteins with deubiquitinase activity, such as TNFAIP3/ A20 or $\beta$-arrestin2, can negatively regulate TRAF6 activity (29). Since we found that bHAf led to a progressive increase in total A20 protein levels in treated slices (Supplemental Figure 6B), we determined whether bHAf-mediated AKT inactivation was related to A20 recruitment into the TRIF-TRAF6 complex. We isolated the TRAF6 complex by co-IP. We confirmed that the isolated complex contained the components of the signalosome by probing it with TRIF and Pellino1/2 antibodies. Pellino proteins are another member of the TRIF complex that can regulate TLR signaling (30). Western blot analysis demonstrated that both TRIF and Pellino1/2 were isolated as part of the TRAF6 co-IP, but we did not detect A2O as part of the signaling complex (Supplemental
Figure 6C). These observations suggest that although bHAf influences A2O induction, bHAf does not appear to play a role in A2O recruitment to the TRIF complex. We next investigated whether bHAf influenced the recruitment of $\beta$-arrestin2, another negative TRAF6 regulator, to the TRIF complex. bHAf did not change the total $\beta$-arrestin 2 level but led to transient recruitment of $\beta$-arrestin2 to the TRIF-TRAF6 complex (Figure 5F). Collectively, these results support that $\beta$-arrestin2 is recruited to the TRIF-TRAF6 complex to attenuate AKT signaling in response to bHAf.

bHAf signaling regulates $O L$ lineage maturation at the preOL stage via FoxO3. To determine downstream targets of AKT activation, we first analyzed mTOR signaling, which plays a role in regulating OPC maturation and myelination (24). However, there were no observed changes in mTOR phosphorylation status in response to bHAf that would suggest a role in bHAf signaling (Supplemental Figure 6D). Prior observations suggested that AKT inactivation results in GSK3 $\beta$ activation and that GSK3 $\beta$ inhibition enhances OPC maturation (31). We thus hypothesized that if bHAf blocked OPC maturation via GSK3 $\beta$ activation, treatment with a GSK3 $\beta$ kinase inhibitor should promote OPC maturation. bHAf treatment attenuated GSK3 $\beta$ Ser9 phosphorylation, which suggested activation of the kinase (Supplemental Figure 6E). We next attempted to block the effect of bHAf with pharmacological agents that target Ser9 phosphorylation of GSK3 $\beta$ to inhibit the kinase. However, the GSK3 $\beta$ inhibitors 6BIO (Supplemental Figure 6F) and LiCl (data not shown) failed to promote OPC maturation, which supported that GSK3 $\beta$ is not part of the bHAf signaling pathway.

We next explored a function for FoxO TFs, known AKT targets, which maintain tissue homeostasis and are sensitive to cellular stress (32). Studies with $\mathrm{FoxO}^{-/-}$mice found that FoxO3 induces a program that prevents premature differentiation of oligodendrocytes by repressing myelination genes (32). Given that AKT phosphorylation was attenuated in chronic WMI until P10, we hypothesized that if FoxO3 contributed to myelination delay, FoxO3-labeled OPCs should be enriched in WM lesions arising from neonatal rat $\mathrm{H}-\mathrm{I}$. We determined whether FoxO3 displayed enhanced nuclear localization to PDGFR $\alpha$-labeled OPCs in WMI at P4-P10. Little FoxO3 staining was detected in control WM at P7 (Figure 6A). However, robust FoxO3 staining localized to WM lesions but did not colocalize with PDGFR $\alpha$-labeled OPCs (Figure 6A). In chronic human neonatal WMI (17) and MS (33), myelination failure arises when OL lineage progression is blocked at the late OPC (preOL) stage. Since we previously found that preOLs also undergo arrested differentiation in chronic rodent WMI, we determined whether FoxO3 localized to preOLs visualized with the anti-O4 antibody. In controls at P7, a low level of cytoplasmic FoxO3 staining was detected that did not colocalize with O4-labeled cells (Figure 6B). However, many O4-labeled cells displayed robust FoxO3 nuclear label in WM lesions (Figure 6B). Hence, maturation arrest of preOLs in chronic WMI coincided with stage-dependent expression of FoxO3 in preOLs.

FoxO3 expression was similarly increased in slice cultures treated with MDa HA for 21 days (Figure 6C) or bHAf for 24 hours (Figure 6D and Supplemental Figure 7A). When we costained slices with FoxO3 and the OL lineage marker and TF Olig2, few FoxO3 ${ }^{+} \mathrm{Olig}^{+}$colabeled cells were visualized in untreated slices, but both MDa HA (Figure 6C) and bHAf (Figure 6D) increased the 
A
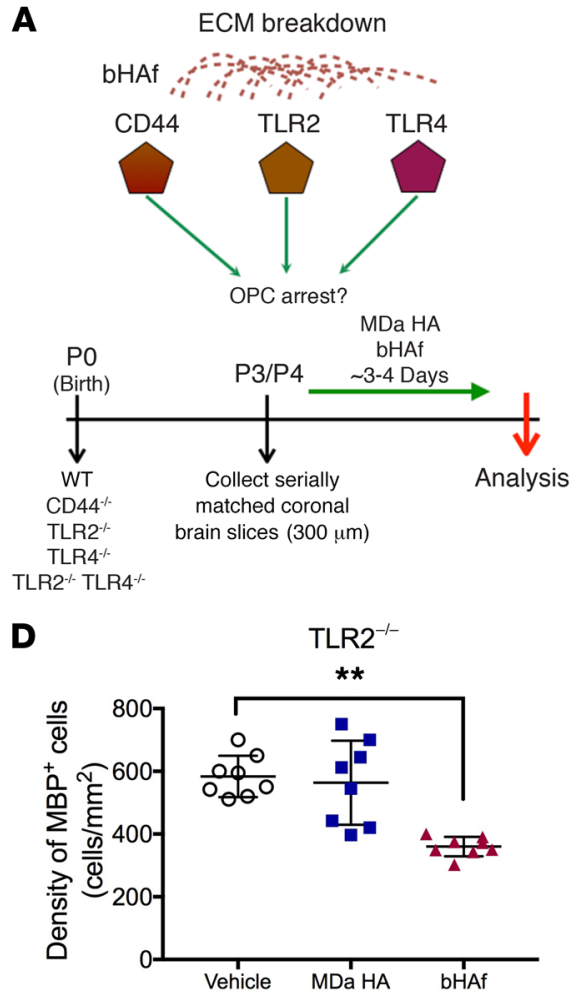

B

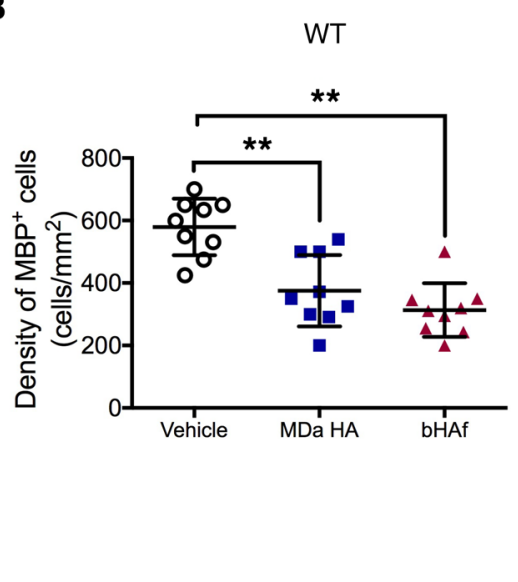

$\mathbf{E}$

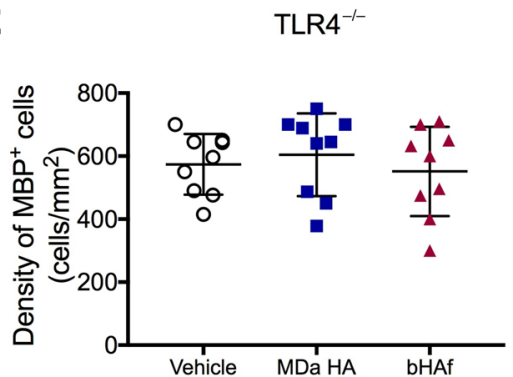

C

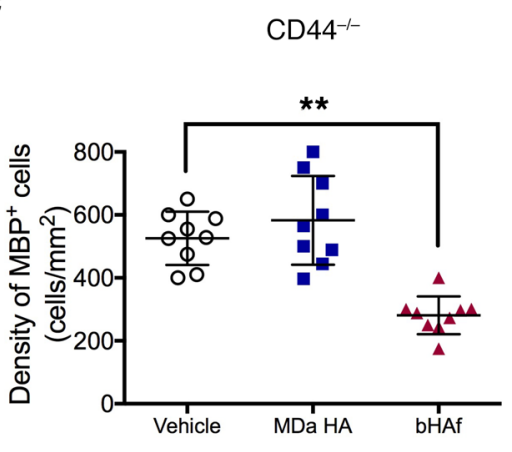

$\mathbf{F}$

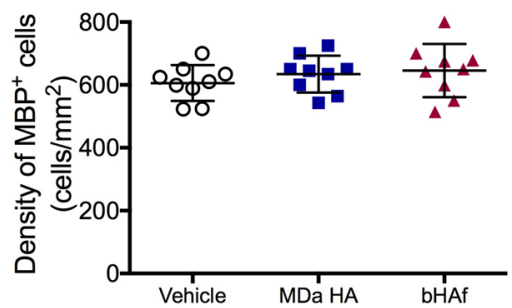

Figure 4. bHAf signals via TLR4 but not TLR2 or CD44 to mediate OPC maturation arrest. (A) Strategy to analyze roles of CD44, TLR2, and TLR4 in myelination failure. Quantification of $\mathrm{MBP}^{+} \mathrm{OL}$ density in corpus callosum from murine forebrain slices treated with PBS (vehicle), MDa HA (2,000 kDa; $50 \mathrm{nM}$ ), or bHAf (210 kDa; $100 \mathrm{nM}$ ). (B) Treatment of slices from WT animals is compared against slices from mice with the following backgrounds: (C) CD44 $^{-/-}$, (D) TLR2 $2^{-1-}$, (E) TLR4 ${ }^{-/-}$, and (F) TLR2 ${ }^{-/-}$TLR4 $^{-/-}$. B-F: $n=3$ animals (P3/P4 mouse pups) per condition for each genotype from separate litters; 3 slices analyzed/treatment condition/animal, 9 slices total. ${ }^{* *} P<0.001$ by ANOVA; mean \pm SD.

number of Olig2 $2^{+}$cells that colocalized with FoxO3. As previously described (32), we also observed some FoxO3 staining that did not localize to OL lineage cells (Figure 6C and Supplemental Figure $7 \mathrm{~A})$, which suggested that variable expression of $\mathrm{FoxO} 3$ may occur in different cell types in chronic WMI. Hence, bHAf regulates the expression and cellular distribution of FoxO3 in OL lineage cells during chronic WMI.

Activation of AKT inactivates and restrains $\mathrm{FoxO}_{3}$ in the cytosol (34). To test whether bHAf-mediated desensitization of AKT is instructive in regulating FoxO3 activity in OPCs, we overexpressed a mutant construct of FoxO3 (FoxO3TM) that lacks AKT phosphorylation sites and causes preferential nuclear localization (34). Primary OPCs were cotransfected with monomeric red fluorescent protein (mRFP; to label transfected cells) and either WT FoxO3 (FoxO3WT) or mutant (FoxO3TM) plasmids and induced to differentiate with or without bHAf (Figure 6E). Treatment with bHAf led to an approximately $50 \%$ decrease in $\mathrm{MBP}^{+}$cells compared with untreated controls. Remarkably, the maturation of OPCs that were transfected with FoxO3TM but not treated with bHAf did not differ significantly from that of untreated control cells. Notably, treatment of FoxO3TM-transfected cells with bHAf had an additive effect, significantly decreasing OL maturation when compared with bHAf-treated control cells, which indicated that nuclear localization of FoxO3 is not sufficient to block OPC maturation and bHAf stimulation is instructive and required. Importantly, when OPCs were transfected with WT FoxO3, there was no significant amplification of bHAf treatment when compared with controls treated with bHAf (Figure 6E). Hence, bHAf is required for downstream activation of FoxO3, since transfection with FoxO3TM alone only mildly blocked OPC maturation and required additional stimulation by bHAf to promote significant OPC maturation arrest.

FoxO3 transcriptional regulation by bHAf involves Olig2 and the chromatin-remodeling factor Brg1. Since our results support that bHAf regulates FoxO3 expression, we examined bHAf-dependent interactions between FoxO3 and other transcriptional regulators of OPC differentiation. Olig2 can recruit Brg1, the ATPase subunit of switch/sucrose non-fermentable (SWI/SNF) chromatin-remodeling protein complexes, to gene enhancers that drive OL differentiation (15). Furthermore, we recently demonstrated that Brg1 regulates OPC specification and OL maturation by controlling expression of genes involved in early OL versus neuronal differentiation (16). In Caenorhabditis elegans, a homolog of FoxO3, FOXO interacts with the SWI/SNF complex to regulate life span (35). We therefore tested the possibility that FoxO3, like other genes that influence OL differentiation, is regulated by SWI/SNF chromatin-remodeling factors recruited by Olig2 and that $\mathrm{FoxO} 3$ interacts with myelin gene promoters regulated by Olig2 and SWI/SNF complexes. We were unable to demonstrate any protein-protein interactions between $\mathrm{Brg} 1$ and $\mathrm{FoxO} 3$ by co-IP (data not shown). However, when we performed ChIP assays, we found that under conditions that promote OPC proliferation or differentiation in primary culture, Brg1 and Olig2 were both bound to the same 
A

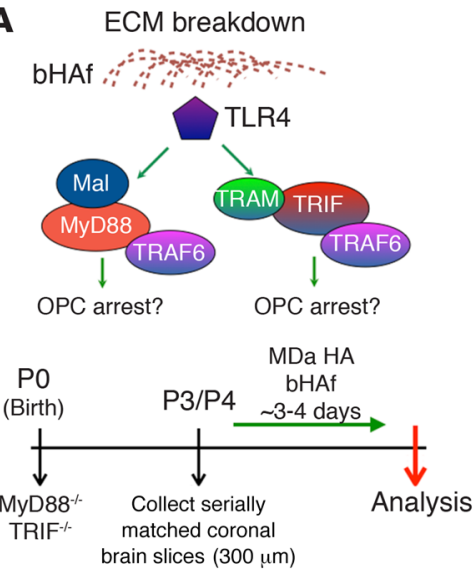

D

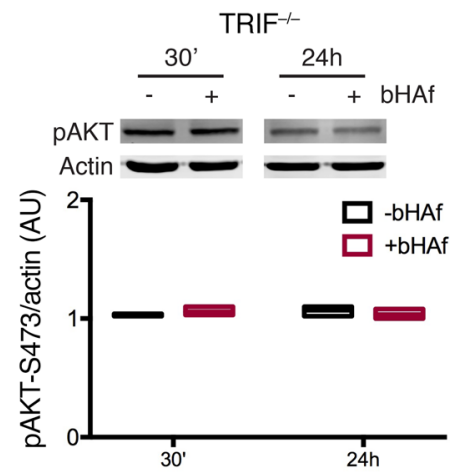

B

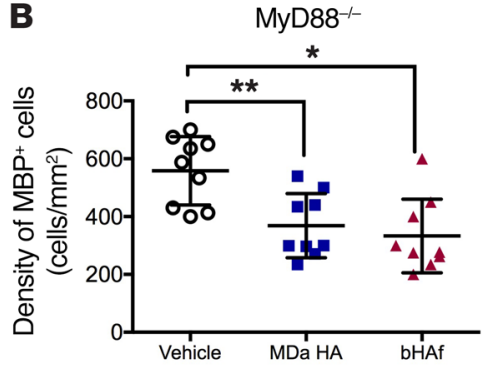

E

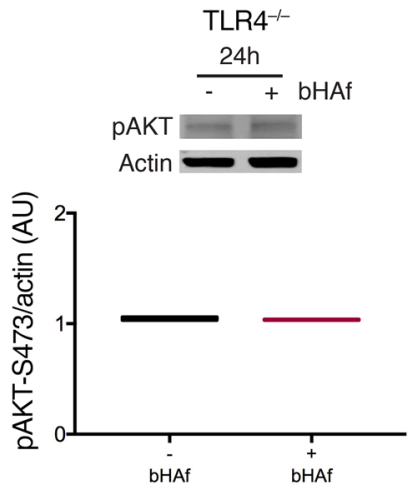

C

$\mathrm{TRIF}^{--}$

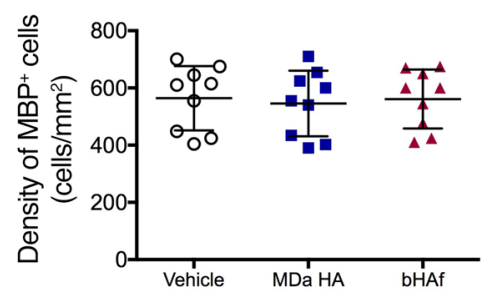

$\mathbf{F}$
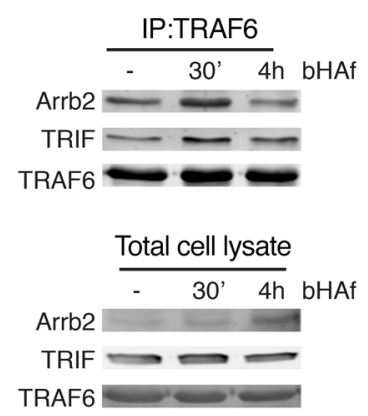

Figure 5. bHAf signals via TRIF to regulate AKT. (A) Strategy to define roles for MyD88 and TRIF in myelination failure. (B) OPC maturation was blocked in MyD88 ${ }^{-1-}$ murine slices treated with MDa HA $(50 \mathrm{nM})$ or bHAf $(100 \mathrm{nM})$. (C) TRIF ${ }^{-/-}$slices treated with MDa or bHAf did not differ from those treated with vehicle (PBS). ( $D$ and E) bHAf-induced AKT phosphorylation (pAKT-S473) is TRIF and TLR4 dependent. Representative blots and quantification from studies with TRIF ${ }^{-1-}$ (D) or TLR4 ${ }^{-/-}$(E) animals. (F) $\beta$-Arrestin2 (Arrb2) is rapidly recruited to the TRIF-TRAF6 complex following bHAf treatment. Lysates were prepared from rat slices treated with or without bHAf $(100 \mathrm{nM})$ at the indicated times and analyzed by IP using TRAF6 antibody. Representative blots were probed with TRIF, TRAF6, and $\beta$-arrestin2 antibodies: IP (upper panel); total cell lysate (lower panel). B and C: $n=3-4$ mice (P3/P4) per condition for each genotype from separate litters; 3 slices/animal/treatment condition; 9-12 slices total. $\mathbf{D}$ and $\mathbf{E}: n=2$ mice (P3/P4) per condition for each genotype from separate litters; 2 slices/animal/treatment condition. $\mathbf{F}: n=2$ experiments from 2 separate litters; 2 slices/condition. ${ }^{*} P<0.05$ by ANOVA; ${ }^{* *} P<0.001$ by ANOVA; mean \pm SD (B and $\mathbf{C})$ and mean \pm SEM (D and $\mathbf{E})$.

region of the FoxO3 promoter (Figure 6F). This interaction was disrupted by treatment of OPCs with bHAf, which displaced Brg1 from the FoxO3 promoter.

Since the transcription of several myelin genes, including $M B P$, were inhibited in OPCs treated with bHAf (Supplemental Figure 7B), we hypothesized that FoxO3 might interact with the $M B P$ promoter to influence Olig2-Brg1-dependent $M B P$ transcription. To begin to define the mechanism by which FoxO3 regulates $M B P$ and other myelin genes, we analyzed interactions among Brg1, Olig2, and FoxO3 at the MBP promoter in OPCs. Under conditions that promote OPC proliferation, Brg1, Olig2, and FoxO3 were found to interact at the same regions of the MBP promoter, but under pro-differentiation conditions $\mathrm{FoxO} 3$ was displaced from the promoter (Figure 6G), consistent with the notion that FoxO3 acts as a transcriptional repressor of $M B P$. However, when pro-differentiation conditions were combined with bHAf treatment, Brg1 and Olig2 were also displaced together with $\mathrm{FoxO} 3$ from the MBP promoter (Figure 6, G and H). These results are consistent with the hypothesis that $\mathrm{FoxO} 3$ represses myelin gene expression at least in part by disrupting transcriptional activation mediated by SWI/SNF complexes recruited to myelin promoters by Olig2. These data further indicate that bHAf inhibits OPC maturation through a mechanism whereby FoxO3, Brg1, and Olig2 are all dissociated from myelin gene promoters under conditions that would otherwise favor OL differentiation.

FoxO3 expression is markedly increased in oligodendroglia in chronic human WMI from preterm neonates and adults with MS. Since our findings supported that nuclear FoxO3 localization is related to myelination failure, we determined whether this signaling paradigm was conserved in chronic human WMI and if FoxO3 could be used as a surrogate readout of tolerance-like dysregulation of human OPC maturation. We first determined the patterns of FoxO3 expression in autopsy samples from 4 individuals with preterm WMI where we previously demonstrated that arrested preOL maturation occurred in lesions enriched in reactive astrocytes and HA (17). Numerous FoxO3-labeled cells defined the boundaries of diffuse WMI (Figure 7A, left panel), but FoxO3 staining was markedly reduced in normal-appearing WM adjacent to the lesion (Figure 7A, middle). In WM lesions (Figure 7A, right), diffuse FoxO3 staining localized to numerous reactive astrocytes and nuclei. 

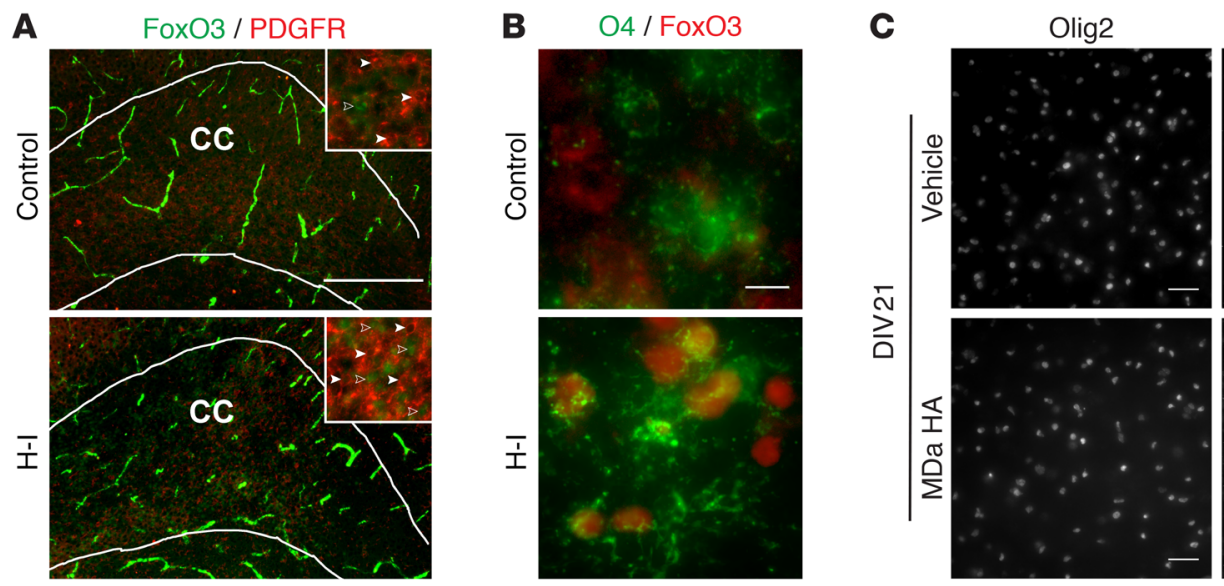

FoxO3

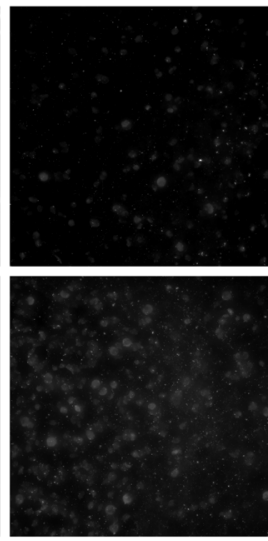

Olig2/FoxO3

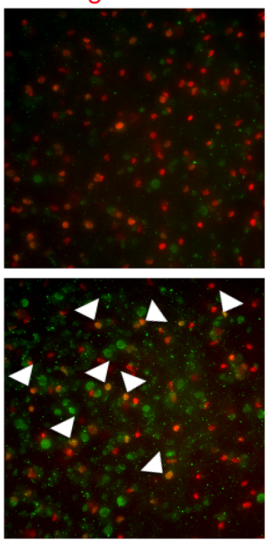

D

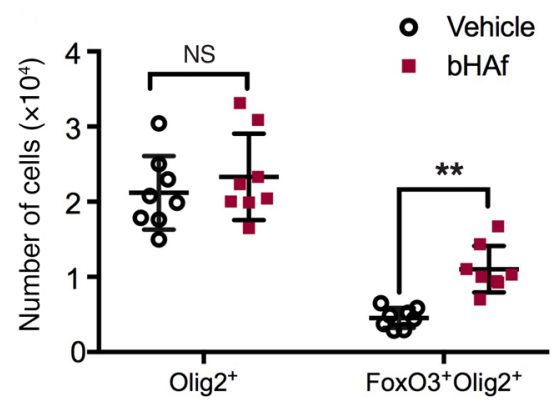

$\begin{array}{ll}\begin{array}{c}\text { Proliferating } \\ \text { OPCs }\end{array} & \begin{array}{c}\text { Differentiating } \\ \text { OPCs -bHAf }\end{array}\end{array}$

FoxO3 promoter

G

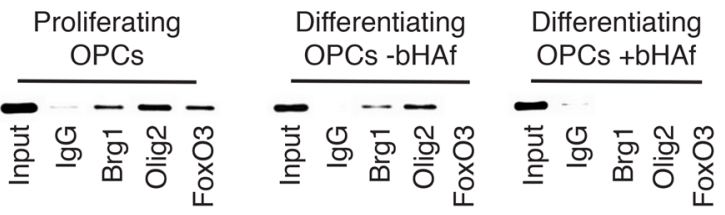
MBP promoter
E
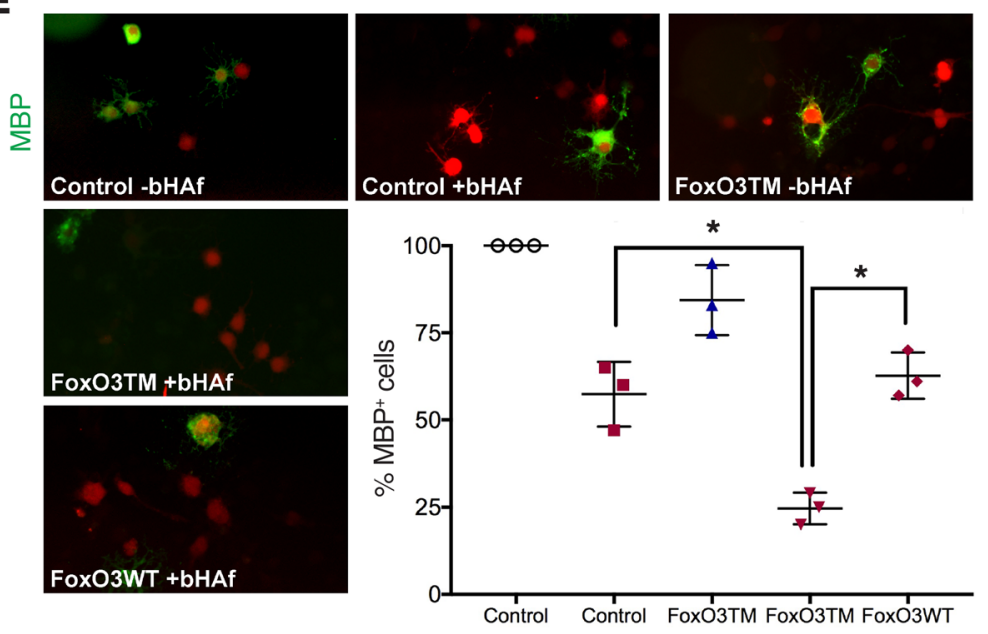

bHAf -
H

OL differentiation factors/ Pro-myelination factors
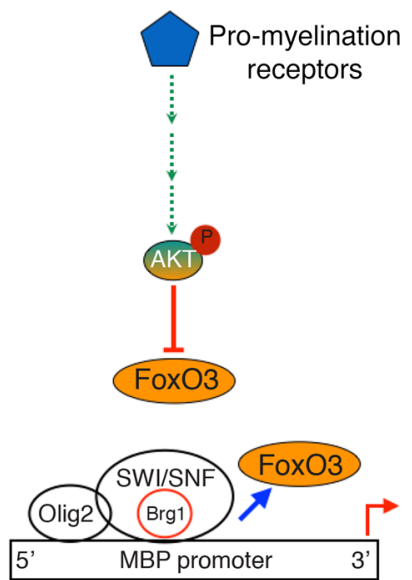

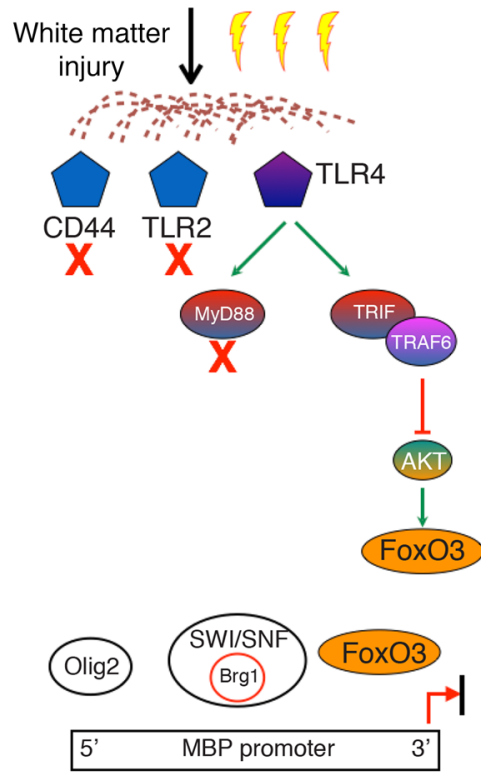


Figure 6. bHAf signaling regulates $\mathrm{OL}$ lineage maturation at the preOL stage via Fox03. (A) Elevated FoxO3 expression in chronic WMI in corpus callosum 4 days after $\mathrm{H}-\mathrm{I}$. Insets: The number of FoxO3-labeled cells (green; open arrowheads) is increased in WMI, but they do not colocalize with PDGFR $\alpha$-labeled progenitors (red; filled arrowheads). (B) Nuclear localization of FoxO3 was detected 4 days after $\mathrm{H}$-I in 04-labeled cells in WMI but not controls. (C) Rat slices treated 21 days with or without MDa HA ( $50 \mathrm{nM})$. MDa HA significantly increased Olig2 ${ }^{+} \mathrm{FoxO3}^{+}$cells in corpus callosum (white arrowheads) versus PBS controls. (D) Total Olig2 ${ }^{+}$and Olig2 ${ }^{+} \mathrm{FoxO3}^{+}$cells in the entire corpus callosum from rat slices treated 24 hours with or without bHAf (100 nM). (E) Primary mouse OPC cultures were cotransfected with the indicated constructs and mRFP (red) and induced to differentiate for 4 days with or without bHAf (500 $\mathrm{nM}$ ). OPCs expressing Fox03TM displayed a significant decrease in the total percentage of MBP-labeled OLs versus cells expressing Fox03WT or control plasmids. ( $F$ and $\mathbf{C}$ ) Molecular interactions among FoxO3, Brg1, and Olig2 in proliferating and differentiating OPCs and in response to bHAf (500 nM). ChIP assay defining Brg1 and Olig2 interactions with the $\mathrm{FoxO}$ promoter (F). ChIP assay defining FoxO3, Brg1, and Olig2 interactions with the MBP promoter (C). (H) Working model depicts that FoxO3 associates with SWI/SNF and Brg1 at the MBP promoter in proliferating OPCs but dissociates from the promoter (blue arrow) when pro-myelination signals induce OPC differentiation, consistent with $\mathrm{FoxO} 3$ repression of MBP transcription. When bHAf inactivates AKT and drives FoxO3 nuclear localization, SWI/SNF and Olig2 dissociate from the MBP promoter, blocking its transcription. $\mathbf{A}$ and $\mathbf{B}: n=2$ control and $n=3 \mathrm{H}-\mathrm{I}$ animals. C: $n=2$ animals/condition from 2 litters; 6 slices total, 3 slices/condition/animal. D: $n=3$ animals/condition; 3 litters; 9 slices total, 3 slices analyzed/condition/animal. E: $n=3$ separate experiments and culture preparations. $\mathbf{F}$ and $\mathbf{G}: n=2$ separate experiments from separate culture preparations. ${ }^{*} P<0.05$ by Student's $t$ test $(\mathbf{E}) ;{ }^{*} P<0.001$ by Student's $t$ test (D); mean SEM. Scale bars: $300 \mu \mathrm{m}, \mathbf{A}$ (insets: original magnification, $\times 40) ; 10 \mu \mathrm{m}, \mathbf{B} ; 40 \mu \mathrm{m}, \mathbf{C}$. Original magnification, $\times 20$ (E).

We analyzed FoxO3 expression in autopsy samples from 4 individuals with chronic MS plaques. To assess FoxO3 expression in uninjured WM relative to demyelinated or partially remyelinated MS plaques, we costained for the myelin marker MBP. MBP staining defined both the chronic active (Figure 7B, left panel) and active remodeling (Figure $7 \mathrm{C}$, left) MS plaques. In chronic active plaques, FoxO3-labeled nuclei were diffusely localized at the plaque borders, where sparse myelination and myelin debris were present (Figure 7B, middle), as well as in the demyelinated core (Figure 7B, right). FoxO3 expression in reactive astrocytes demarcated the plaque borders and was also detected in the lesion core (Figure 7B, right, and Supplemental Figure 8A). Within active remodeling plaques, FoxO3 localized to numerous nuclei interspersed near sparse myelination (Figure 7C, middle). However, in heavily myelinated WM distal to the plaque, few FoxO3-labeled nuclei were detected (Figure 7C, right).

Since FoxO3 localized to the nuclei of OPCs that displayed arrested maturation in rat $\mathrm{H}-\mathrm{I}$ lesions (36) (Figure 6B), we next determined whether FoxO3-labeled nuclei in human lesions also localized to the OL lineage. To visualize OL lineage cells in paraffin-embedded neonatal tissue, we employed the pan-OL lineage marker Olig2, which robustly identified lesions where an expanded pool of OL progenitors had arrested maturation (17). Numerous Olig2 ${ }^{+}$nuclei colocalized with FoxO3 in neonatal WM lesions (Figure 7D and Supplemental Figure 8B). Similarly, in chronic active MS plaques, numerous Olig2 ${ }^{+}$nuclei colocalized with FoxO3 (Figure 7D and Supplemental Figure 8C). Hence, oligodendroglial cells that expressed nuclear FoxO3 were present both in chronic preterm human WMI and MS plaques, and the persistence of these cells may contribute to myelination disturbances after WMI.

\section{Discussion}

IT induction in the innate immune system following repeated exposure to DAMPs is an evolutionarily conserved mechanism to prevent excessive inflammation in response to infection or tissue injury (2). During early inflammation, depolymerization of MDa HA attenuates macrophage/monocyte activation and blocks inflammation in several contexts (1). Neonatal WMI also triggers early and persistent ECM breakdown in chronic lesions where delayed myelination occurs. We succeeded in isolating an endogenous activity in neonatal WMI lysates that cleaves exogenously added MDa HA into a range of fragment sizes. We defined a role for specific HAf that induce a tolerance-like state that attenuates AKT activation and promotes myelination failure. Disrupted OPC maturation was selectively mediated via an approximately 200-kDa bHAf and was reversed when MDa HA depolymerization was blocked by a hyaluronidase inhibitor. bHAf signaled via a noncanonical TLR4/TRIF pathway to dysregulate AKT/FoxO3 signaling. bHAf downregulated myelin gene expression through a mechanism that includes dissociation of a SWI/SNF chromatin remodeling factor and Olig2 from myelin gene promoters. Hence, our findings support the action of an IT-like mechanism in response to WMI whereby chronic desensitization of AKT attenuates pro-myelination signals, which promotes an OPC niche at the expense of myelination (Figure 6H).

During mammalian embryonic CNS development, hyaluronidase expression coincides with low MDa HA accumulation, but postnatally MDa HA accumulates whereas hyaluronidase expression is undetectable (18). WMI was accompanied by a pronounced reduction in MDa HA. Decreased MDa HA may be mediated by HA synthases that are induced to generate only small HAf (37). Our analysis of neonatal $\mathrm{H}-\mathrm{I}$ lesions supports that depletion of MDa HA was related to enhanced hyaluronidase expression. From in vitro models of adult WMI, hyaluronidase $\mathrm{PH} 20$ was proposed to play a role in delaying remyelination mediated by MDa HA (6, 7). A recent report has questioned this role of PH2O (19). We did not detect $\mathrm{PH} 2 \mathrm{O}$ expression in normal or injured neonatal brain, which may suggest that $\mathrm{PH} 2 \mathrm{O}$ expression is maturation dependent or transient. In response to neonatal WMI, we observed decreased expression of Hyal1, a hyaluronidase involved in secretion of small-sized HAf from endocytosed HA (18) and increased Tmem2, a recently identified hyaluronidase that generates intermediate-sized HAf (20). This prompted us to explore the hypothesis that intermediate-sized HAf block OPC maturation. We screened highly pure endotoxin-free HAf sizes and identified a bHAf of approximately $200 \mathrm{kDa}$.

Emerging studies have identified unique conformational properties of the approximately $200-\mathrm{kDa}$ bHAf that may account for its selective dysregulation of myelination after WMI (38). As a polymer in solution, MDa HA largely exists as a stiff random coil. However, multiple studies found that as HA size decreases it transitions from a random coil to a rod-like conformation (38). This size-dependent conformational transition occurs in the range of 100-300 kDa, and HAf of more than $300 \mathrm{kDa}$ may essentially have the same conformation as MDa HA (38). That the effects of bHAf we observed were not attenuated in competition studies with other HAf sizes further suggests unique biological properties at this size range. The conformation of bHAf may also induce binding of 


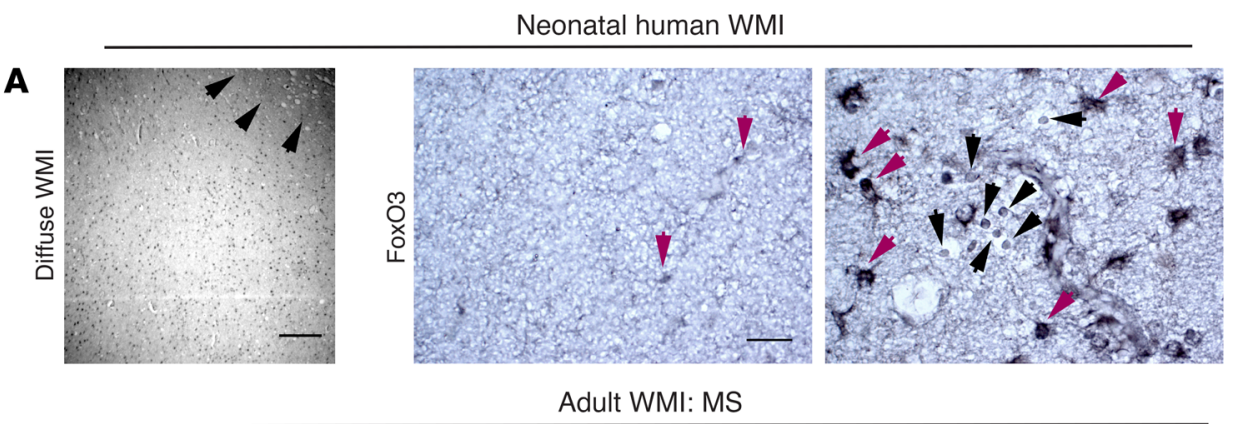

B
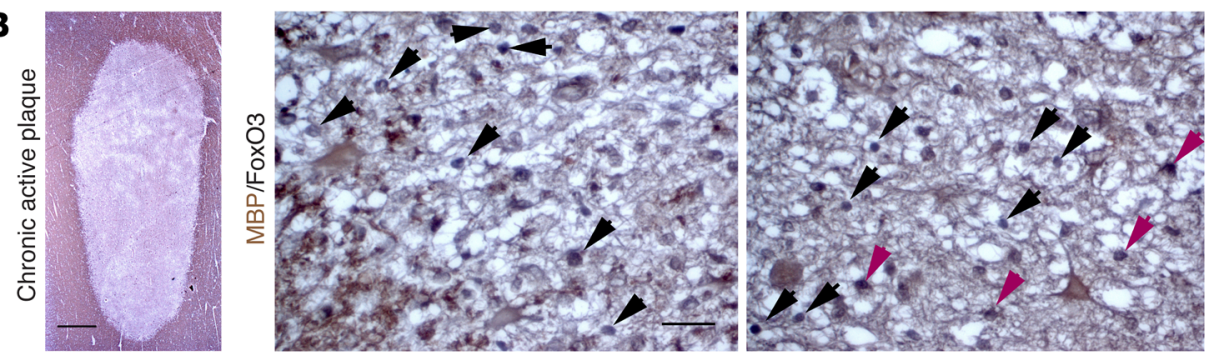

C
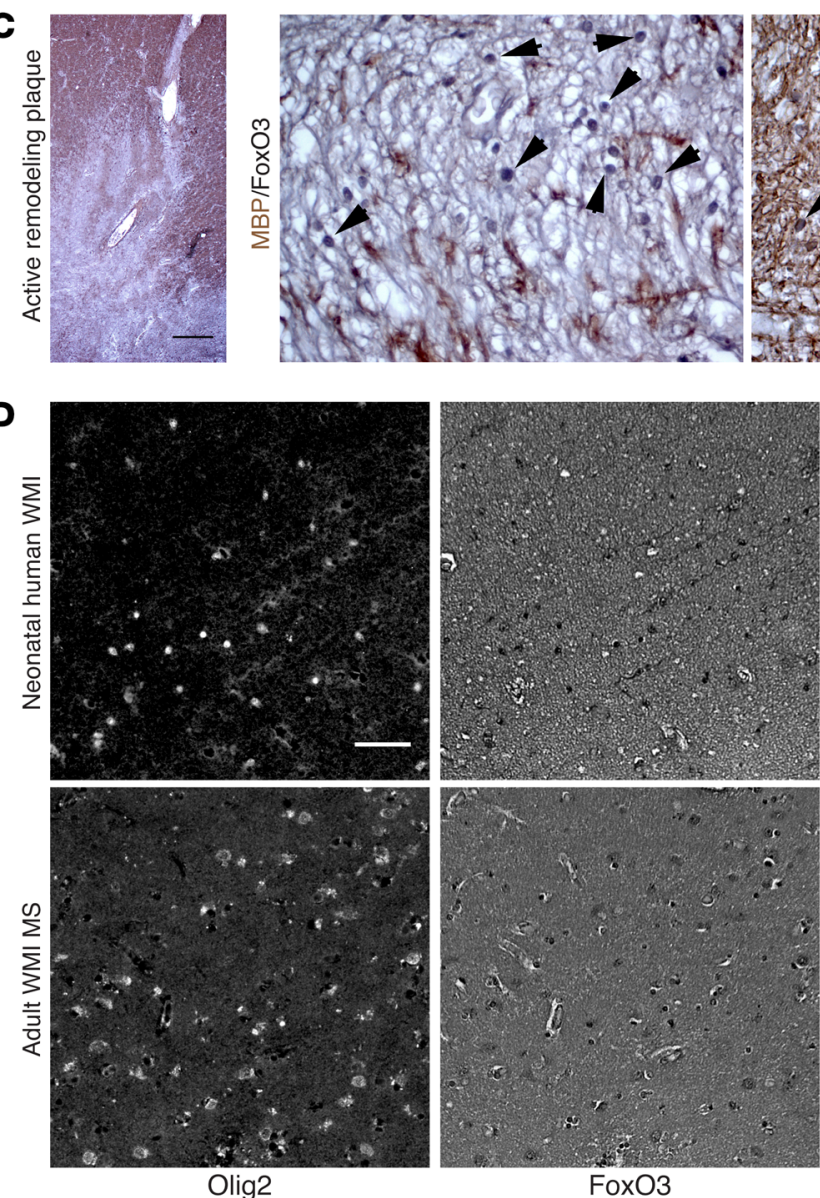

FoxO3
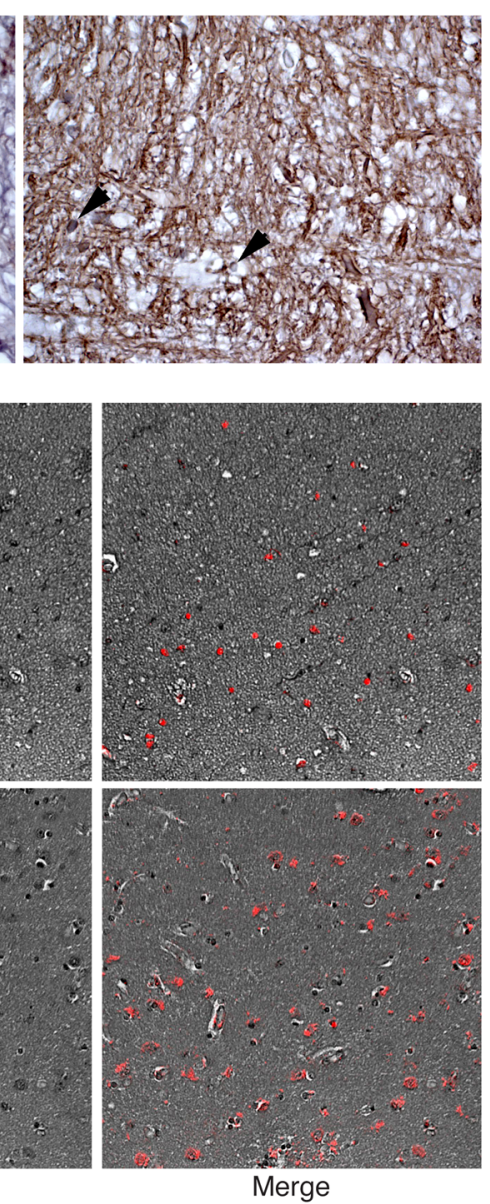

Figure 7. Fox03 expression is markedly increased in oligodendroglia in chronic human WMI from preterm neonates and adults with MS. Robust $\mathrm{Fox} 03$ expression localized differentially to the cytoplasm of astrocytes and nuclei of Olig2 ${ }^{+}$oligodendroglia in chronic human WMI. (A) Left: Low-power image of preterm WMI (Supplemental Table 1A; case 3). Diffuse FoxO3 staining defined boundaries of this large lesion between the cortical mantle (arrowheads) and the lateral ventricle (not shown). Middle: Rare Fox03-labeled cells (red arrowheads) in normal-appearing WM adjacent to the lesion. Right: $\mathrm{FoxO}$ localized to numerous reactive astrocytes (red arrowheads) and nuclei (black arrowheads) in the lesion. (B) FoxO3 expression in a chronic active MS plaque (Supplemental Table 1B; case 1). Left: Low-power image of a chronic active plaque stained for $\mathrm{FoxO}$ and $\mathrm{MBP}$ with markedly reduced MBP staining in the core versus the peri-plaque. Middle: Detail of the plaque edge shows scattered MBP-labeled myelin debris (brown) and numerous FoxO3-labeled nuclei (black arrowheads). Right: Within the plaque core, no myelination was visualized, but many FoxO3-labeled nuclei (black arrowheads) were interspersed among reactive astrocytes (red arrowheads). (C) FoxO3 expression in an active remodeling MS plaque (Supplemental Table 1B; case 1). Left: Low-power image of the plaque stained for $\mathrm{Fox} \mathrm{O} 3$ and $\mathrm{MBP}$ with scattered islands of remyelination within the lesion. Middle: Numerous FoxO3-labeled nuclei (black arrowheads) localized among sparsely myelinated axons (brown). Right: A heavily myelinated peri-plaque region with rare Fox03-labeled nuclei (black arrowheads). (D) FoxO3-labeled nuclei in a preterm WM lesion (Supplemental Table 1A; case 1) and MS plaques (Supplemental Table 1B; case 3 ) colocalized with the pan $\mathrm{OL}$ lineage marker Olig2. Colocalization of Olig2 fluorescence and $\mathrm{Fox} 03$ colorimetric labeling is indicated in the merge in red. Scale bars: $200 \mu \mathrm{m}, \mathbf{A}$, left; $20 \mu \mathrm{m}, \mathbf{A}$, middle and right; $300 \mu \mathrm{m}, \mathbf{B}$, left; $20 \mu \mathrm{m}$, B and C, middle and right; 300 $\mu \mathrm{m}, \mathbf{C}$, left; $30 \mu \mathrm{m}, \mathbf{D}$. a particular hyaladherin to form a complex that is recruited by a cell-surface or intracellular protein to form a unique signaling complex (38). For example, cell surface CD44 has higher binding affinity for HA in complex with the TSG-6 link module compared with HA alone (39). The formation of such complexes with HAf may explain why it has been technically challenging in multiple tissues to detect endogenous bHAf in vivo. For example, recent studies with human breast milk found it to be highly enriched in HA sizes from 100 to $1,500 \mathrm{kDa}(40)$, which suggested a role for breast milk in regulation of gut innate immune responses (41). Human milk and a $35-\mathrm{kDa}$ HAf similarly regulated gut immune responses, but biochemical analysis did not detect enrichment of 35-kDa HAf in human milk. In studies of bleomycin-induced murine lung fibrosis, an HAf of $540 \mathrm{kDa}$ was initially identified (42). Although an in vitro 
screen of purified HAf later identified a proinflammatory role for a 135-kDa HAf (43), this size was not enriched in murine fibrotic lung tissue (42). Similarly, our in vivo biochemical analysis of $\mathrm{H}$-I lesions found an overall decrease in total MDa HA, but no enrichment of the approximately 200-kDa bHAf.

Studies of noninfectious lung injury, skin lesions, wound healing, and activation of immune dendritic cells have indicated various roles for CD44, TLR2, and TLR4 in mediating HAf actions (8). We identified roles for CD44, TLR2, and TLR4 in regulation of OPC maturation after WMI. When either CD44 or TLR2 was deleted, bHAf but not MDa HA retained activity to block OPC maturation.

However, TLR $4^{-/-}$mice were insensitive to both bHAf and MDa HA. Hence, contributions from CD44 and TLR2 appeared to be required for MDa HA to block OPC maturation, whereas bHAf actions specifically required an interaction with TLR4. Notably, binding of larger-sized HA appears to be stabilized by multivalent interactions with CD44, whereas smaller HAf has distinct binding properties (44). Polydispersed HA of larger sizes in human milk also requires both CD44 and TLR4 to mediate their biological effects, whereas 35-kDa HAf works independently of CD44 (41). Our findings similarly support a primary role for bHAf in mediating OPC maturation arrest via TLR4 independent of CD44 and TLR2. Thus, targeting CD44 and/or TLR2 to prevent MDa HA de-polymerization or TLR4 to prevent bHAf signaling may be an attractive strategy to promote regeneration and repair of neonatal WMI.

In the context of neonatal WMI, TLR4 regulated AKT not but MAPK in a TRIF-dependent manner. Similarly, a prior study of normal myelination or remyelination did not support a role for the IKK/NF- $\mathrm{BB}$ pathway downstream of TLRs (10). Persistent bHAf treatment led to desensitization of AKT, which was not rescued by BDNF. BDNF potently induces AKT activation via the TrkB receptor. Thus, bHAf-mediated desensitization of AKT signaling appears to share characteristics with IT induced by persistent activation of TLRs by endotoxins, which recruits negative regulators to dampen multiple downstream signaling effectors $(1,2,29)$. We found that $\beta$-arrestin2, a known negative regulator of TLR signaling (29), was rapidly recruited to the TRIF-TRAF6 complex, upstream of AKT desensitization induced by bHAf. Attenuation of AKT activation persisted for at least 9 days in vitro, which suggests involvement of other signaling regulators in this pathway. Furthermore, the onset of delayed myelination after neonatal $\mathrm{H}$-I followed a similar time course. That myelination was stimulated in neonatal WMI as AKT phosphorylation normalized suggests that myelination failure may be reversible when bHAf levels decline in chronic lesions. In fact, even after prolonged exposure to MDa HA in primary (4) or slice cultures, arrested OPC maturation was reversible when MDa HA was removed. Since our findings suggest that attenuation of AKT activation is reversibly dependent on ECM remodeling, it may be feasible to design therapeutic strategies to promote myelination by specifically targeting the signaling proteins that are involved in mediating the tolerance-like state induced by bHAf.

Attenuation of AKT activity results in activation of GSK3 $\beta$ kinase and FoxO TFs (34). Our results do not support a role for GSK3 $\beta$ kinase in bHAf-mediated myelination failure and agree with previous studies, using knockin mice, that found that regulation of GSK3 $\beta$ Ser9 phosphorylation appears to have no physiological actions in the nervous system (45). AKT is activated by inhibi- tors of GSK3 $\beta$ Ser9 phosphorylation (e.g., LiCl, 6BIO) (46), which may account for observations that these inhibitors promoted OPC maturation (31).

Several findings supported that bHAf regulates FoxO3 to block preOL maturation. bHAf increased $\mathrm{FoxO}^{+} \mathrm{Olig} 2^{+}$cells in chronic in vitro slices. Moreover, forced expression of FoxO3TM, which preferentially resides in the nucleus, did not block OPC maturation without bHAf treatment, which supports that initiation of bHAf signaling is a requirement for FoxO3 activity. Olig2 and Brg1 both bind to the FoxO3 promoter in a bHAf-dependent manner, which suggests that FoxO3, like other factors that regulate OL differentiation, is transcriptionally regulated by Olig2-recruited SWI/SNF complexes and that bHAf controls FoxO3 transcription by disrupting these interactions. Furthermore, FoxO3 interacts with the $M B P$ promoter in a region where Olig2 and Brg1 also interact, and $\mathrm{FoxO} 3$ is dissociated from the $M B P$ promoter as OPCs differentiate. This suggests a mechanism by which FoxO3 acts to restrict OPC differentiation. Given that FoxO3 co-associates with Brg1 and Olig2 at the $M B P$ promoter, it is possible that FoxO3 blocks $M B P$ transcription by interfering with the activities of SWI/SNF complexes recruited to the promoter by Olig2. Interestingly, our data also show that under differentiation conditions in the presence of bHAf, Brg1 and Olig2 are also dissociated from the $M B P$ promoter, which suggests that bHAf signaling leads to dissociation of Olig2 from the promoter to block transcription.

The actions of nuclear FoxO3 in promoting OPC maturation arrest appeared to be exerted in a maturation-dependent fashion in vivo through selective nuclear localization to preOLs. In contrast, in chronic preterm WMI and MS plaques, cytoplasmic FoxO3 demarcated lesion boundaries by robustly localizing to reactive astrocytes, as a potential marker of chronic ECM remodeling. Although our results support that bHAf blocks preOL maturation via FoxO3, the role of other FoxO isoforms in neonatal WMI is unclear. FoxO TFs appear to exert redundant effects to coordinately regulate diverse pathways to modulate neural stem cell or progenitor cell homeostasis $(32,47)$. In a mouse model of chronic neonatal hypoxia, FoxO1 was shown to promote OPC proliferation (48). It is thus interesting to note that TGF- $\beta$ signaling regulates the timing of myelination by mediating interactions between FoxO1 and Sp1, another TF that regulates OPC maturation (49). Wnt signaling is another negative regulator of OPC maturation during WMI that also downregulates Sp1-mediated transcription (50). However, TGF- $\beta$ signaling does not regulate subcellular distribution of FoxO3 or AKT activation (51), whereas bHAf-mediated AKT desensitization drives FoxO3 activation. Given that activation of FoxO3 initiates a positive feedback loop that promotes transcription of FoxO1 and FoxO4 (52), it will be interesting to determine the epigenetic relationships between different FoxO TFs and Sp1 during chronic WMI and their influence on myelination failure.

Definition of the pathogenetic mechanisms that regulate the generation and actions of bHAf may suggest repair strategies in several neonatal and adult human disorders where WMI results in chronic myelination failure. Persistent signaling by bHAf via the TLR/TRIF pathway results in chronic desensitization of AKT, and FoxO3 activation sustains arrested preOL maturation and myelination failure. Strategies to reverse this tolerance-like state appear to represent a novel approach to promote myelin regeneration. 


\section{Methods}

Pharmacological reagents. Endotoxin-free, pharmaceutical-grade high-molecular-weight HA (MDa HA; $>1.8 \mathrm{MDa} ;<0.1 \mathrm{EU} / \mathrm{mg}$ ) and polydispersed HAf (5-20 kDa, <0.01 EU/mg; and 175-300 kDa, <0.05 $\mathrm{EU} / \mathrm{mg}$ ) were from Lifecore Biomedical. HA of defined narrow-size ranges (monodispersed) were prepared from Lifecore polydispersed HA by SEC fractionation (23). Groups of 3 fractions with similar masses, assessed by SEC-MALS, were pooled from multiple column runs and concentrated, and final HA weight-average molar mass (Mw), $96 \%$ size distribution, concentration, and endotoxin levels were determined: 40-kDa HAf (<0.004 EU/mg), 106-kDa HAf (<0.002 EU/mg), 210-kDa HAf ( $<0.002 \mathrm{EU} / \mathrm{mg})$, and 357-kDa HAf $(<0.002 \mathrm{EU} / \mathrm{mg})$. All HA stocks were made in endotoxin-free PBS. 3-[1-[3-(dimethylamino)propyl]-5-methoxy-1H-indol-3-yl]-4-(1H-indol-3-yl)-1H-pyrrole2,5-dione (Go6983) and (2'Z,3'E)-6-bromoindirubin-3'-oxime (6BIO) were from TOCRIS Bioscience. $\mathrm{LiCl}$ was from MilliporeSigma. BDNF was from PeproTech.

Animals. The following strains were from the Jackson Laborato-

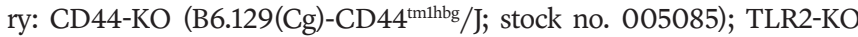
(B6.129-Tlr2 ${ }^{\text {tm1Kir } / J ; ~ s t o c k ~ n o: ~ 004650) ; ~ T L R 4-K O ~(B 6 . B 10 S c N-T l r 4 ~} 4^{\text {lps-del }} /$ JthJ; stock no: 007227); MyD88-KO (B6.129P2(SJL)-Myd88 ${ }^{\text {tm1.1Defr } / J ; ~ s t o c k ~}$ no: 009088); TRIF-KO (C57BL/6J-Ticam1 $1^{\mathrm{Lps} 2} / \mathrm{J}$; stock no: 005037). WT C57BL/6 mice from the Jackson Laboratory served as strain-matched controls. TLR2 ${ }^{-/-}$TLR4 ${ }^{-/-}$mice were provided by Salman Qureshi (McGill University, Montreal, Quebec, Canada) (53). E17 timed-pregnant Sprague-Dawley (SD) rats were from Charles River.

Neonatal H-I. Unilateral cerebral H-I was studied in P3 rats as described previously (36). Untreated true controls and H-I animals survived until P4, P7, P10, P14, or P21. Only brains with grossly visible cerebral pallor or swelling were analyzed by IHC (36) or were rapidly dissected, snap frozen in liquid nitrogen, and stored at $-80^{\circ} \mathrm{C}$ for RNA, protein, and HA analyses. A minimum of 5 brains were analyzed at each time point. Up to 20 animals from 2 separate litters were combined without identifiers for H-I. Studies were repeated at least once for a minimum of 4 litters entered into each analysis.

In vitro hyaluronidase assay and SEC-MALS. For the in vitro hyaluronidase assay, protein lysates were prepared from WM lesions from $\mathrm{P} 4$ rats that underwent $\mathrm{H}-\mathrm{I}$ at $\mathrm{P} 3$. Lysates were prepared in ice-cold $1 \times$ RIPA buffer containing protease inhibitor cocktail (MilliporeSigma). Lysates $(40 \mu \mathrm{g})$ were incubated with MDa HA $(8 \mu \mathrm{g})$ in buffer containing $1 \% \mathrm{TX}-100,150 \mathrm{mM} \mathrm{NaCl}, 100 \mathrm{mM}$ formic acid, $\mathrm{pH} 4.0$, at $37^{\circ} \mathrm{C}$ for 4 hours. Some protein lysates were heat inactivated by boiling for 30 minutes or treated with deferoxamine (10 $\mu \mathrm{M}$; MilliporeSigma) before incubation with MDa HA. Samples were briefly centrifuged, and $10 \mu \mathrm{l}$ of reaction solution was loaded onto $0.5 \%$ agarose gels for electrophoresis for 3 hours at $20 \mathrm{~V}$ at $4^{\circ} \mathrm{C}$. Gels were stained with Stains-all (Polysciences Inc.) and imaged using the Odyssey Infrared system (Li-COR Biosciences).

HA concentration and $M w$ in brain tissues was determined by SECMALS using 2 or 3 PL Aquagel-OH 60, 40, and 20 (Polymer Labs) size exclusion columns in series (7). MALLS analyses of the SEC column eluate was performed in-line using a Dawn DSP Laser Photometer in series with an Optilab DSP Interferometric Refractometer (both from Wyatt Technologies). Data were analyzed using ASTRA version 4.73, with $\mathrm{dn} / \mathrm{dc}$ values of $0.153 \mathrm{ml} / \mathrm{g}$, and first-order Berry fits.

Forebrain slice culture and primary OPC culture. Whole forebrain coronal slices $(300 \mu \mathrm{m})$ were prepared from $\mathrm{PO} / \mathrm{P} 1$ rats or $\mathrm{P} 3 / \mathrm{P} 4$ mice for organotypic cultures as described previously (21). Mouse slices were cultured without penicillin and streptomycin. Half-media changes were done every other day up to DIV7-DIV8 or DIV21 (rat) or DIV3DIV4 (mouse).

Primary OPC cultures were derived from oligospheres (7) or by immunopanning (54). For OPC differentiation experiments, OPCs were plated at $5 \times 10^{4}$ cells per coverslip and differentiated in DMEM/ F12 medium containing 0.1\% BSA plus triiodothyronine (T3; $30 \mathrm{nM}$; MilliporeSigma) and $N$-acetyl-L-cysteine (NAC; MilliporeSigma) for 4 days with or without HAf (500 nM) of indicated sizes. Cells were fixed in ice-cold $4 \%$ paraformaldehyde for 1 hour at room temperature prior to IHC.

Plasmids and cell transfection. HA-FoxO3WT and HA-FoxO3TM plasmids from Addgene were described previously (34). To verify whether FoxO3 influences bHAf signaling, we cotransfected oligosphere-derived OPC cultures $\left(5 \times 10^{4}\right.$ cells $)$ on glass coverslips with mRFP using Lipofectamine 2000 (Invitrogen). The day after transfection, differentiation was induced in the presence or absence of bHAf ( $500 \mathrm{nM}$ ) for 4 days. Cells were fixed at room temperature for 15 minutes in $4 \%$ paraformaldehyde and $4 \%$ sucrose in PBS, permeabilized with $0.1 \%$ TX-100 in PBS for 10 minutes, and visualized with rat anti-MBP antibody (1:500; MAB386; EMD Millipore). Primary antibody was visualized with anti-rat-Alexa 463 (1:500; Invitrogen). Fluorescence images were acquired at $20 \times$ with a cooled CCD camera (Hamamatsu Photonics) attached to a Zeiss Imager.M2 upright microscope. 2,000-3,000 cells were counted per coverslip; 4 coverslips were analyzed per condition from 2-3 independent experiments. Cell counts employed the cell counting plugin in FIJI software.

Real-time PCR analysis. P3 animals were analyzed at 24 hours after H-I. Control and WM lesion tissue were microdissected and resuspended in TRIzol (Life Technologies) to extract total RNA. 250 ng RNA was used to make cDNA using the TaqMan Reverse Transcription Kit (Applied Biosystems). cDNA was diluted 1:1 before realtime PCR (qPCR) experiments (TaqMan Gene Expressions Master Mix, Applied Biosystems). All qPCR probes were FAM-MGB (Thermo Fisher Scientific): Tmem2 (Rn01498397-m1), PH20 (Rn01500727-m1; Rn01500728-m1; Rn00588656-m1), Hyal-1 (Rn02133715-S1), Hyal-2 (Rn01521340-g1),Has1(Rn00597231-m1),Has2 (Rn00565774-m1), Has3 (Rn00597204-m1), MBP (Rn01399619-m1), Plp (Rn01410490-m1), and Mog (Rn00575354-m1). 18S RNA (Rn03928990-g1) was the endogenous control. Tissue was screened for WMI by analyzing changes in GFAP (Rn01253033-m1) expression. Only H-I samples with 4- to 10-fold changes in GFAP expression versus controls were analyzed in duplicate for HAS and Hyal transcripts using ABI 7300 System software. Gene expression is presented as relative quantity or fold change versus control and calculated using the $2^{-\Delta \Delta \mathrm{Ct}}$ method. Statistical analysis was performed on the $\Delta \mathrm{Ct}$ values for each gene.

Co-IP and Western blotting. For Co-IP(s), total protein extracts were prepared in lysis buffer (1\% TX-100, 10\% glycerol, $1 \mathrm{mM} \beta$-glycerol-P, $150 \mathrm{mM} \mathrm{NaCl}, 10 \mathrm{mM} \mathrm{NaF}, 50 \mathrm{mM}$ Tris, $\mathrm{pH}$ 7.4) supplemented with complete protease inhibitor cocktail (MilliporeSigma) and complete phosphatase inhibitor cocktail (Roche). Homogenates were centrifuged $(15,000 \mathrm{~g}, 10$ minutes). $500 \mu \mathrm{g}$ of supernatant total protein lysate was incubated with primary antibodies for 3 hours, followed by 1-hour incubation with Protein A/G Sepharose beads (Invitrogen) at $4^{\circ} \mathrm{C}$. Proteins were washed 3 times from the beads with lysis buffer before SDSPAGE. See complete unedited blots in the supplemental material. 
Antibodies were from the following sources: polyclonal rabbit AKT (1:1,000; C67E7; catalog 4691), A2O (1:1,000; D13H3; catalog 5630), phospho-AKT T-308 (1:1,000; catalog 9275), phospho-AKT S473 (1:1,000; catalog 9271), phospho-ERK1/2 (1:1,000; catalog 9102), phospho-mTOR 2448 (1:1,000; D9C2; catalog 5536), phospho-GSK3 $\beta$ Ser9 (1:500; 5B3; catalog 9323) and mouse monoclonal actin (1:4,000; 8H10D10; catalog 3700) antibodies were from Cell Signaling Technology; monoclonal mouse TRIF (1:200; E-7; catalog sc-514384), TRAF6 (1:200; D10; catalog sc-8409), $\beta$-arrestin2 (1:200; H-9; catalog sc-13140), and rabbit polyclonal Pellino1/2 (1:200; H-105; catalog sc-67025) antibodies were from Santa Cruz Biotechnology Inc. Equivalent protein amounts were analyzed by SDS-PAGE and transferred to an Immobilon-FL membrane. Western blot detection employed IR700 or IR800 or a special IR800-conjugated (incapable of detecting IgG) secondary antibody (Rockland Inc.). The pixel band intensity was analyzed with the Odyssey Infrared system (Li-COR Biosciences).

ChIP assay. Mouse OPCs were grown in proliferation or differentiation medium (7) and treated with vehicle (PBS) or bHAf (500 nM), followed by treatment with $1 \%$ formaldehyde $\left(10\right.$ minutes; $\left.37^{\circ} \mathrm{C}\right)$. Cells were washed 3 times with ice-cold PBS containing a protease inhibitor cocktail (MilliporeSigma), lysed, and chromatin sheared to generate fragments of different sizes $(14,16)$. ChIP assays were performed as described previously (16). For preclearing and pull-downs, we used Protein A Sepharose (GE Healthcare) preblocked with yeast transfer RNA and BSA. The antibodies used were rabbit anti-Olig2 (catalog AB9610; Millipore), rabbit anti-Brg1 (D1Q7F; catalog 49360; Cell Signaling Technology), rabbit anti-FoxO3 (D19A7; catalog 12829; Cell Signaling Technology), and rabbit IgG (catalog 2729; Cell Signaling Technology). PCR was performed using Phusion Hot Start II High Fidelity DNA Polymerase and the following primers: for MBP, forward primer 5'-AGAGGGAATGGACTTGCTGC, reverse primer 5'-GCGGTCGTTTGACAAAAGGT; for FoxO3, forward primer 5'-CGCAGTGGTGTGTTGTCTGA, reverse primer: 5'-CGGCAGCAGCACAAAGTTAT.

IHC. Antibodies used were as follows: mouse anti-MBP (1:500; SMI-99; NE1019; Calbiochem), rat anti-MBP (1:500; MAB386; EMD Millipore), mouse anti-GFAP (1:500; MAB360; EMD Millipore), mouse anti-O4 (1:500; from Steven Pfeiffer, University of Connecticut Health Center, Farmington, Connecticut, USA), rabbit anti-PDGFR $\alpha$ (1:500; from William Stallcup, Sanford Burnham Prebys Medical Discovery Institute, La Jolla, California, USA), mouse anti-CNPase (1:500; SMI-91; Covance), mouse antiCASPR/paranodin (1:250; K65/35; NeuroMab), mouse anti-NF-H (1:1,000; SMI312; BioLegend), rabbit anti-caspase-3 (1:200; 9662; Cell Signaling Technology), mouse anti-FoxO3 C-terminus (1:100; from Anne Brunet, Stanford University, Stanford, California, USA), rabbit anti-FoxO3 (1:100; NB100-614; Novus Biologicals), goat anti-Olig2 (1:100; AF2418; R\&D Systems), and mouse anti-Olig2 (1:500; John Alberta, Dana-Farber Cancer Institute, Boston, Massachusetts, USA). HA was visualized with biotinylated HA-binding protein (bHABP; 1:200; 385911; EMD Millipore). Selectivity of HABP staining was confirmed with controls that excluded HABP or secondary antibody (Supplemental Figure1A). Antigen retrieval was employed for all antibodies ( $0.01 \mathrm{M}$ citrate buffer $\mathrm{pH} 6.0,85^{\circ} \mathrm{C}, 15$ minutes). All corresponding secondary antibodies were from Jackson ImmunoResearch Laboratories Inc. Nuclei were counterstained with DAPI (Invitrogen). No-primary control studies for all antibodies exhibited no specific staining.
Stereology. Cell counts of slice cultures were performed using a Stereo Investigator stereology system (MBF Bioscience). Within the $10 \times$-defined boundaries of the entire corpus callosum (defined by DAPI staining), $\mathrm{MBP}^{+}$, Olig2 ${ }^{+}$, and $\mathrm{FoxO}^{+}$double-labeled cells were estimated using the optical fractionator probe (grid size, $300 \times 400$ $\mu \mathrm{m}$; counting frame, $40 \times 40 \mu \mathrm{m}$; z-depth, $40 \mu \mathrm{m}$ ) at $40 \times$ magnification in a minimum of 30 randomly selected WM fields per slice. Slice thickness was measured at each counting site. Cell density $\left(\mathrm{mm}^{2}\right)$ was calculated by the formula (cell counts/[number of fields $\times$ counting frame area $\left.\left.\left(\mathrm{mm}^{2}\right)\right]\right)$. Estimates of MBP-labeled cells were also obtained using the meander scan function at $20 \times$.

To estimate WM and cortex MBP area fractions, we assessed WMI using an unbiased approach where lesions were not specifically selected. Montages were generated from frontal tissue sections fluorescently stained for MBP. Montages were captured at $10 \times$ magnification, and MBP staining was estimated using rolling-ball background subtraction with a 150-pixel radius. A pixel-intensity histogram was generated for each ROI using Image $(\mathrm{NIH})$ and exported to R (R Project for Statistical Computing), and area fractions were obtained as described previously (17).

Human pathology. We studied 2 cohorts of human autopsy brains with either chronic diffuse preterm WMI or chronic stages of WMI in adult MS. Nine tissue blocks were retrospectively collected from archival cases (formalin-fixed and paraffin-embedded). The 4 tissue blocks with preterm WMI were previously described in Table 1 of ref. 17 and correspond to cases 7, 12, 13, and 17, with ages at birth of 32, 29, 25, and 26 weeks, respectively. Tissue blocks selected for the adult MS cohort had chronic active or chronic remodeling ("shadow") plaques, as previously described (ref. 7 and see Supplemental Table 1).

Statistics. Statistical analysis was performed using Prism 7 statistical software (GraphPad Software). Comparisons between 2 groups were made by 2 -tailed Student's $t$ test. One-way ANOVA followed by Dunnett's multiple comparisons test was used to determine statistical significance for more than 2 groups. $P<0.05$ was considered statistically significant. All experiments were repeated at least twice and performed with several independent biological samples, as indicated in the figure legends.

Study approval. All animal procedures were approved by the OHSU IACUC (protocol IP00000837) according to the NIH Guide for the Care and Use of Laboratory Animals. All neonatal human WMI samples were obtained after full IRB review and approval from Children's and Women's Hospital, University of British Columbia (Vancouver, British Columbia, Canada) with the assistance of Glenda Hendson in the Department of Pathology. All pathological diagnoses were independently confirmed by G. Hendson and Marjorie Grafe. All MS tissue blocks were obtained with the assistance of C. Dirk Keene, Department of Pathology, University of Washington, Seattle, Washington, USA. Tissues were obtained with the approval of the human subject committee of the University of Washington.

\section{Author contributions}

TS, BE, DLM, PHW, LSS, and SAB were responsible for conception and design of the studies. TS, PD, DS, MH, and WS acquired and analyzed data from rodent H-I studies, primary OPCs, and in vitro slice culture for myelination status, gene expression, and actions of HAf. JMD and XG acquired and analyzed rodent H-I time course data to quantify myelination recovery. FB acquired and analyzed all data for promoter expression studies in primary OPCs. BB acquired and analyzed SEC-MALS data from rodent 
H-I lesions; BB and ENH generated the endotoxin-free monodispersed HAf by SEC fractionation. TS, XG, and SAB analyzed the human postmortem samples

\section{Acknowledgments}

This work was supported by the National Institute of Neurological Disorders and Stroke (NSO54044; NSO45737 to SAB), the National Institute on Aging (AG-31892 to SAB and LSS), an NIH Directors award for the operation of the Oregon National Primate Research Center (P51OD01109), the American Stroke Association (grant-inaid 11GRNT7510072 to SAB), the Congressionally Directed Medical Research Programs (MS160144 to LSS), and the National Multiple
Sclerosis Society (RG 4843A5/1 to LSS). We are grateful to Susan Aicher and the OHSU EM core for ultrastructural studies supported by NIH grant P30NS061800. We are grateful to Mary Stenzel-Poore, Susan Stevens, and Dale Fortin for their invaluable discussions regarding mechanisms of IT and signaling and to Marjorie Grafe for valuable advice and review of human neuropathology specimens. TS was supported by a Huebner Family Postdoctoral Fellowship.

Address correspondence to: Stephen A. Back, Oregon Health and Science University, Department of Pediatrics, Division of Pediatric Neuroscience, 3181 S.W. Sam Jackson Park Road, Portland, Oregon 97239-3098, USA. Phone: 503.494.0906; Email: backs@ohsu.edu.
1. Biswas SK, Lopez-Collazo E. Endotoxin tolerance: new mechanisms, molecules and clinical significance. Trends Immunol. 2009;30(10):475-487.

2. Morris MC, Gilliam EA, Li L. Innate immune programing by endotoxin and its pathological consequences. Front Immunol. 2014;5:680.

3. Oohashi T, Edamatsu M, Bekku Y, Carulli D. The hyaluronan and proteoglycan link proteins: Organizers of the brain extracellular matrix and key molecules for neuronal function and plasticity. Exp Neurol. 2015;274(Pt B):134-144.

4. Back SA, et al. Hyaluronan accumulates in demyelinated lesions and inhibits oligodendrocyte progenitor maturation. Nat Med. 2005;11(9):966-972.

5. Gallo V, Deneen B. Glial development: the crossroads of regeneration and repair in the CNS. Neuron. 2014;83(2):283-308.

6. Sloane JA, Batt C, Ma Y, Harris ZM, Trapp B, Vartanian T. Hyaluronan blocks oligodendrocyte progenitor maturation and remyelination through TLR2. Proc Natl Acad Sci U S A. 2010;107(25):11555-11560.

7. Preston M, et al. Digestion products of the PH2O hyaluronidase inhibit remyelination. Ann Neurol. 2013;73(2):266-280.

8. Jiang D, Liang J, Noble PW. Hyaluronan as an immune regulator in human diseases. Physiol Rev. 2011;91(1):221-264.

9. Taylor DL, et al. Attenuation of proliferation in oligodendrocyte precursor cells by activated microglia. J Neurosci Res. 2010;88(8):1632-1644.

10. Raasch J, et al. IkappaB kinase 2 determines oligodendrocyte loss by non-cell-autonomous activation of NF-kappaB in the central nervous system. Brain. 2011;134(Pt 4):1184-1198.

11. Dong Y, et al. Endotoxin free hyaluronan and hyaluronan fragments do not stimulate TNF- $\alpha$, interleukin-12 or upregulate co-stimulatory molecules in dendritic cells or macrophages. Sci Rep. 2016;6:36928.

12. Back SA, Rosenberg PA. Pathophysiology of glia in perinatal white matter injury. Glia. 2014;62(11):1790-1815.

13. Flores AI, et al. Constitutively active Akt induces enhanced myelination in the CNS. J Neurosci. 2008;28(28):7174-7183.

14. Banine F, et al. SWI/SNF chromatin-remodeling factors induce changes in DNA methylation to promote transcriptional activation. Cancer Res. 2005;65(9):3542-3547.

15. Yu Y, et al. Olig2 targets chromatin remodelers to enhancers to initiate oligodendrocyte differentiation. Cell. 2013;152(1-2):248-261.

16. Matsumoto S, et al. Brg1 directly regulates Olig2 transcription and is required for oligodendrocyte progenitor cell specification. Dev Biol. 2016;413(2):173-187.

17. Buser JR, et al. Arrested preoligodendrocyte maturation contributes to myelination failure in premature infants. Ann Neurol. 2012;71(1):93-109.

18. Stern R, Jedrzejas MJ. Hyaluronidases: their genomics, structures, and mechanisms of action. Chem Rev. 2006;106(3):818-839.

19. Marella M, et al. $\mathrm{PH} 20$ is not expressed in murine CNS and oligodendrocyte precursor cells. Ann Clin Transl Neurol. 2017;4(3):191-211.

20. Yamamoto H, Tobisawa Y, Inubushi T, Irie F, Ohyama C, Yamaguchi Y. A mammalian homolog of the zebrafish transmembrane protein 2 (TMEM2) is the long-sought-after cell-surface hyaluronidase. J Biol Chem. 2017;292(18):7304-7313.

21. Dean JM, et al. An organotypic slice culture model of chronic white matter injury with maturation arrest of oligodendrocyte progenitors. $\mathrm{Mol}$ Neurodegener. 2011;6:46.

22. Botzki A, et al. L-Ascorbic acid 6-hexadecanoate, a potent hyaluronidase inhibitor. X-ray structure and molecular modeling of enzyme-inhibitor complexes. J Biol Chem. 2004;279(44):45990-45997.

23. Pandey MS, Baggenstoss BA, Washburn J, Harris EN, Weigel PH. The hyaluronan receptor for endocytosis (HARE) activates NF- $\kappa \mathrm{B}$-mediated gene expression in response to 40-400-kDa, but not smaller or larger, hyaluronans. J Biol Chem. 2013;288(20):14068-14079.

24. Bercury KK, Macklin WB. Dynamics and mechanisms of CNS myelination. Dev Cell. 2015;32(4):447-458.

25. Han $\mathrm{BH}$, et al. BDNF blocks caspase-3 activation in neonatal hypoxia-ischemia. Neurobiol Dis. 2000;7(1):38-53.

26. Fortin DA, et al. Brain-derived neurotrophic factor activation of CaM-kinase kinase via transient receptor potential canonical channels induces the translation and synaptic incorporation of GluA1-containing calcium-permeable AMPA receptors. J Neurosci. 2012;32(24):8127-8137.

27. Harada H, Takahashi M. CD44-dependent intracellular and extracellular catabolism of hyaluronic acid by hyaluronidase-1 and -2. J Biol Chem . 2007;282(8):5597-5607.

28. Rolls A, et al. Toll-like receptors modulate adult hippocampal neurogenesis. Nat Cell Biol.
2007;9(9):1081-1088.

29. Kondo T, Kawai T, Akira S. Dissecting negative regulation of Toll-like receptor signaling. Trends Immunol. 2012;33(9):449-458.

30. Moynagh PN. The roles of Pellino E3 ubiquitin ligases in immunity. Nat Rev Immunol. 2014;14(2):122-131.

31. Azim K, Butt AM. GSK3 $\beta$ negatively regulates oligodendrocyte differentiation and myelination in vivo. Glia. 2011;59(4):540-553.

32. Renault VM, et al. FoxO3 regulates neural stem cell homeostasis. Cell Stem Cell. 2009;5(5):527-539.

33. Chang A, Tourtellotte WW, Rudick R, Trapp BD. Premyelinating oligodendrocytes in chronic lesions of multiple sclerosis. N Engl J Med. 2002;346(3):165-173.

34. Brunet A, et al. Akt promotes cell survival by phosphorylating and inhibiting a Forkhead transcription factor. Cell. 1999;96(6):857-868.

35. Riedel CG, et al. DAF-16 employs the chromatin remodeller SWI/SNF to promote stress resistance and longevity. Nat Cell Biol. 2013;15(5):491-501.

36. Segovia KN, et al. Arrested oligodendrocyte lineage maturation in chronic perinatal white matter injury. Ann Neurol. 2008;63(4):520-530.

37. Baggenstoss BA, Harris EN, Washburn JL, Medina AP, Nguyen L, Weigel PH. Hyaluronan synthase control of synthesis rate and hyaluronan product size are independent functions differentially affected by mutations in a conserved tandem B-X7-B motif. Glycobiology. 2017;27(2):154-164.

38. Weigel PH, Baggenstoss BA. What is special about $200 \mathrm{kDa}$ hyaluronan that activates hyaluronan receptor signaling? Glycobiology. 2017;27(9):868-877.

39. Lesley J, et al. TSG-6 modulates the interaction between hyaluronan and cell surface CD44. J Biol Chem. 2004;279(24):25745-25754.

40. Yuan H, Amin R, Ye X, de la Motte CA, Cowman MK. Determination of hyaluronan molecular mass distribution in human breast milk. Anal Biochem. 2015;474:78-88.

41. Hill DR, et al. Human milk hyaluronan enhances innate defense of the intestinal epithelium. J Biol Chem. 2013;288(40):29090-29104.

42. Teder $\mathrm{P}$, et al. Resolution of lung inflammation by CD44. Science. 2002;296(5565):155-158.

43. Jiang D, et al. Regulation of lung injury and repair by Toll-like receptors and hyaluronan. Nat Med. 2005;11(11):1173-1179.

44. Wolny PM, et al. Analysis of CD44-hyaluronan 
interactions in an artificial membrane system: insights into the distinct binding properties of high and low molecular weight hyaluronan. J Biol Chem. 2010;285(39):30170-30180.

45. Hur EM, Zhou FQ. GSK3 signalling in neural development. Nat Rev Neurosci. 2010;11(8):539-551.

46. Ding VW, Chen RH, McCormick F. Differential regulation of glycogen synthase kinase 3 beta by insulin and Wnt signaling. J Biol Chem . 2000;275(42):32475-32481.

47. Paik JH, et al. FoxOs cooperatively regulate diverse pathways governing neural stem cell homeostasis. Cell Stem Cell. 2009;5(5):540-553. 48. Jablonska B, et al. Oligodendrocyte regen- eration after neonatal hypoxia requires FoxO1-mediated p27Kip1 expression. J Neurosci. 2012;32(42):14775-14793.

49. Palazuelos J, Klingener M, Aguirre A. TGF $\beta$ signaling regulates the timing of CNS myelination by modulating oligodendrocyte progenitor cell cycle exit through SMAD3/4/FoxO1/Sp1. J Neurosci. 2014;34(23):7917-7930.

50. Fancy SP, et al. Parallel states of pathological Wnt signaling in neonatal brain injury and colon cancer. Nat Neurosci. 2014;17(4):506-512.

51. Seoane J, Le HV, Shen L, Anderson SA, Massagué J. Integration of Smad and forkhead pathways in the control of neuroepithelial and glioblastoma cell proliferation. Cell. 2004;117(2):211-223.

52. Essaghir A, Dif N, Marbehant CY, Coffer PJ, Demoulin JB. The transcription of FOXO genes is stimulated by FOXO3 and repressed by growth factors. J Biol Chem. 2009;284(16):10334-10342.

53. Paun A, Fox J, Balloy V, Chignard M, Qureshi ST, Haston CK. Combined Tlr2 and Tlr4 deficiency increases radiation-induced pulmonary fibrosis in mice. Int J Radiat Oncol Biol Phys. 2010;77(4):1198-1205.

54. Dugas JC, Tai YC, Speed TP, Ngai J, Barres BA. Functional genomic analysis of oligodendrocyte differentiation. J Neurosci. 2006;26(43):10967-10983. 